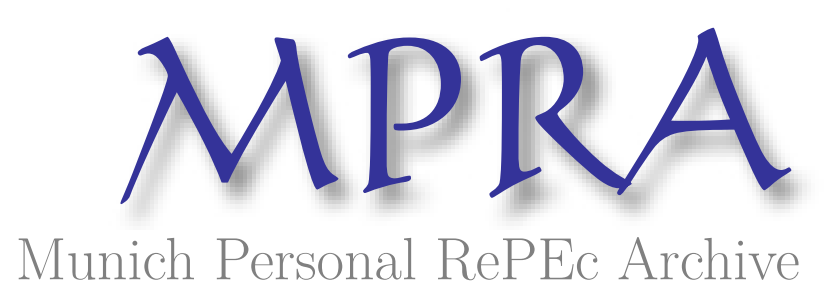

\title{
Choosing a transport contract over multiple periods
}

Brusset, Xavier

Center of Excellence in Supply Chain Management, Louvain School of Management, Université catholique de Louvain

9 January 2009

Online at https://mpra.ub.uni-muenchen.de/18392/

MPRA Paper No. 18392, posted 11 Nov 2009 00:07 UTC 
Choosing a transport contract over multiple periods

\title{
Xavier Brusset
}

Centre of Excellence in Supply Chain Management,

Louvain School of Management,

Université Catholique de Louvain,

Place des Doyens, 1 B-1348 Louvain la Neuve, Belgium

E-mail: xavier.brusset@uclouvain.be

\begin{abstract}
We offer a shipper and a carrier the choice among three contracts in which to frame their relationship. Both can also take recourse in the transport spot market. Demand and price on the spot market are dependent exogenous stochastic processes. We model the outcome of this endogenous choice of contract. The results, given in closed form, are different from those presented in the literature. Using numeric instances, we show how a choice is made and which contract would be preferred. Comparison on the variance of the economic returns are offered. The conclusions are applicable when the carrier is not capacity constrained.
\end{abstract}

Keywords: transport; stochastic process; MPC; minimum purchase commitment; quantity flexibility; relational contract.

Reference to this paper should be made as follows: Brusset, X. (2009) 'Choosing a transport contract over multiple periods', Int. J. Logistics Systems and Management, Vol. 5, Nos. 3/4, pp.273-322.

Biographical notes: Xavier Brusset is in the course of finishing his Doctoral Thesis (October 2008). He is part of a team of researchers financed by the Walloon Region for a two year euro 15 million research project. He founded in 2000 a company in Argentina to set up an internet platform of logistic services to the transport industry using the Software as a Service (SaaS) model. He graduated as a MBA from the Ecole Supérieure de Commerce de Paris, France.

\section{Introduction}

We observe that in transport, very frequently manufacturers have relationships with several carriers. Often, no formal contracts back them up. The price of transport is per unit and independent of volume. The manufacturer may at times take recourse to a list of other carriers when his first choice of carrier cannot pick up a load. The price in this case may fluctuate and at times, there may be no available transport capacity. In other cases, the manufacturer prefers to tie his business to a carrier by a formal contract using some form of commitment of quantities to be carried in each period of 
time (a Minimum Purchase Commitment or MPC) or over several periods (a Quantity Flexibility Commitment or QFC). How do the manufacturer and carrier decide on which contract is best and with which parameters? We attempt to solve this problem when both carrier and shipper will repeatedly renew their contracting arrangements. It is the point of this paper to show that previous literature on the subject of contracts in transport and in supply chain management may not be pointing to optimal contract or contract parameters when carrier and shipper have the choice of contract.

Three types of contracts are compared in profit maximising functions. To the preceding two, we add the single price per unit for all volumes often characterised as the Price only Relational Contract (PRC).

In Section 2 we review the relevant literature, in Section 3 we explain the model, in Section 4 we explain how a contract is chosen. In Section 5 numerical examples are presented before concluding in Section 6.

\section{Literature review}

Papers making endogenous the contract selection mechanism are few. Generally, when modelling interactions between a buyer and a supplier with imperfect information, the contract being chosen or mechanism being designed is viewed as exogenous. In effect, in such a setting, games will yield a certain outcome which may be optimal. However, how does this optimal behave when both players have to choose between such a contract and other contracts? Jackson and Wilkie (2005) models a contract in which players can write 'side contracts' where they adjust the original contract to affect the outcome of the game and its equilibrium. These side contracts result from a rich choice of possible 'side payments' that each player can make to the other. In effect, this means that both players have the choice of contract. This type of 'theoretical' contract does not really apply to situations observed in transport. We know from Lagunoff (1992) that fully endogenous mechanism selection (selection of institutional arrangements) is non-vacuous when the class of mechanisms is restricted at each stage of a process. ${ }^{1}$

In supply chain management literature, in the presence of multi-period stochastic demand Martínez-de-Albéniz and Simchi-Levi (2005) addresses procurement through a diversity of contracts and spot market buying. Martínez-de-Albéniz and Simchi-Levi devise a portfolio of contracts to replenish stock from a variety of suppliers. Minimum commitment and quantity flexibility contracts are shown to be reducible to portfolios of options. But why would suppliers agree to sell options and not insist on firm commitments, since they will incur the cost of reserving capacity? Also, Corbett et al. (2004) shows that with three types of contracts, deterministic demand and asymmetric information, information has greater value with two-part contracts then with one-part contract. Wu et al. $(2001,2002,2005)$ study the contracting arrangements in energy sector between a producer and several buyers. The buyer can take recourse in the spot market at the daily spot price. The decision variables are the reservation price and execution price per unit of capacity. The spot market enables the buyer to satisfy her needs in case the contractual arrangement is not enough. A mixture of spot procurement and long-term contract procurement are shown to be the optimal for a supply chain. Unhappily, these models do not really apply to transport as spot prices are modelled as varying in direct function with demand. 
Spinler and Huchzermeier (2006) builds upon Wu et al. (2002) and is one of the few who study spot market price and demand as two random variables. In a game theoretic setting, the seller acts as Stackelberg leader by bidding an option two-part tariff menu consisting in a reservation price and an execution fee ahead of revelation of demand. This problem is solved as a dynamic programming problem. This option on capacity problem bears close resemblance to the setting which we will use in the present paper as the MPC contract. There are two differences. One is that here the buyer must buy from the seller the contracted capacity, even when the spot price is lower than the variable fee that she pays for each effectively bought unit. The other difference is that in Spinler and Huchzermeier (2006), the buyer can decide not to buy if the spot price is too high, which is not the case here. The present paper assumes that the buyer always serves exogenous demand: she relies on the spot market.

Tsay et al. (1999) list Quantity Flexibility Clauses (QFC) and Minimum Purchase Commitments (MPC) providing justification of their usefulness in coordinating a supply chain and which will be considered as proven here. This classification assumes that each contract is exogenously given.

Cachon and Lariviere (2001), Özalp and Wei (2006) and Brusset and Temme (2005) draw attention to the information imbalance prevalent in most supply chains which has special consequences when the supplier is capacity constrained and can decide on contract compliance level. The conclusion which is used here is: the supply chain is better coordinated under assumption of asymmetric information when both players set up a firm commitment for capacity with a lump sum payment.

Tomlin (2003) builds upon and refines Cachon and Lariviere (2001) by comparing the contract choices open to manufacturer (buyer) and supplier in the case of variable demand so that the supplier is coordinated into investing into the proper capacity to respond to stochastic demand. When processing costs at the supplier are non-linear, it is shown that three forms of contract dominate the linear-price contract and enhance coordination compared to the centrally organised case: quantity-premium, firm-commitment and option when the supplier has positive capacity cost (our case). In the game considered here, as opposed to Tomlin's game, the preliminary capacity investment is excluded as it is a sunk investment, ${ }^{2}$ information about demand is supposed to be asymmetric and there is a back-up source.

Tsay and Lovejoy (1999) and Tsay (1999) describe in detail the contract model which inspires us here: the total minimum quantity commitment where a buyer guarantees that his cumulative orders across all periods in the planning horizon will exceed a specified minimum quantity. In the present paper an incentive is added that ensures coordination in the supply chain in the form of a penalty as described in Chen (2004).

Since the two players operate in a repeated game setting, the general working of the negotiations with alternated offers before a contract is signed are inspired from Plambeck and Taylor (2005, 2007). First observed in Macauley (1963), the supporting arguments for the informal PRC enforced by reputational concerns and between parties who interact repeatedly are presented more thoroughly as 'relational contract' in Baker et al. (2001, 2002). Namely, repeated interaction introduces dynamics in relationships that influence the costliness and effectiveness of actions in the future. These repeated interactions facilitate the use of informal agreements not sustained by court system, but by the ongoing value of the relationship. 


\section{Transport contract model}

\subsection{Repeated game with bivariate output demand and input price}

The shipper (which, to avoid confusion, will be termed a 'she') has to satisfy transport for her product and to do so must buy transport services either from a carrier through an ex ante arrangement or from a spot market. Remember that transport, as a service, cannot be stocked and has no salvage value if unused. One unit of transport capacity used corresponds to one unit of product carried. The shipper's residual demand not covered by the long-term contract is resolved by buying additional transport capacity from the carrier at the going spot market price. The spot market is the place where excess demands and excess transport capacities meet and transactions take place at prices which may fluctuate and which usually clears the market. The remaining unused capacities are considered as lost and the remaining transport demand is rescheduled for the following day. It is considered in this model that both shippers and carriers influence this spot market price by their action. A spot market exists as soon as there are demands and capacities which are standard enough to be interchangeable. ${ }^{3}$

\subsection{Asymmetric information about demand and utility functions}

We model asymmetric information as in Cachon and Lariviere (2001). Demand $X$ is part of a family of scaled distributions (see Lariviere, 1999, for a definition of scaled distributions) which is characterised by a factor $\mu_{X}>0$ such that for all $\mu_{X}^{\prime} \leq \mu_{X}$, $F_{X}\left(x \mid \mu_{X}^{\prime}\right)>F_{X}\left(x \mid \mu_{X}\right)$ for $x>0$ and $F_{X}\left(0 \mid \mu_{X}^{\prime}\right) \geq F_{X}\left(0 \mid \mu_{X}\right)$. Only the shipper knows the parameter $\mu_{X}$ and can estimate the transport capacity she will require from it. Thus, the demand is stochastically increasing in $\mu_{X}$. The shipper is also private of her utility function. The carrier is private to his own costs and other opportunities, he needs credible information about the capacity that the shipper will need so as not to commit more transport capacity than necessary, even though we have not modelled any limit to his existing capacity. We assume that the highest possible demand that the shipper may conceivably ask to be transported is limited to $Q_{\mathrm{High}}$. This limit is assumed to be common knowledge and to be less than the transport capacity available to the carrier (either from his fleet or from known third party carriers operating in the spot market which can be subcontracted). On the other hand, the information about the spot market price, mean, variance and distribution is assumed to be freely observable by both.

The spot market is not well organised so information is sparse and costly (in difference to the models developed in Spinler and Huchzermeier (2006), Kleindorfer and $\mathrm{Wu}$ (2003), Seifert et al. (2004), Wu and Kleindorfer (2003)). To access this information, a player who wishes to use this spot market incurs a fixed cost $I$ per period. The carrier has only one technology at his disposal and hence his production facility is homogeneous, which simplifies fixed cost attribution. He has the installed capacity in place to satisfy the shipper's demand, but this capacity is not expandable at short notice. His capacity is shared among all his customers, minimising risk of capacity under-usage by pooling it.

Some mention must be made here of the fact that the carrier appears to be modelled as enjoying unlimited capacity. In reality, this type of carrier usually is a third-party logistics operator who just out-sources transport requirements to a vast number of 
owner-operated trucks or he is a trucking company who calls up extra capacity from sub-contractors or other trucking companies when faced by demand in excess of his available fleet capacity.

This type of carrier is known to exist in road and sea transport and have been looked into in a number of transport research papers. The model presented here can easily be extended to railway and even air freight because it is always possible to contract extra capacity from other companies in the same industry. This type of carrier relies on the existence of a spot market among carriers where loads and excess capacity can be offered.

Contrary to most models in supply chain management literature but along with Spinler and Huchzermeier (2006), no storage, holding or shortage costs are incurred for the transport capacity by the carrier. The supply lead time is assumed to be zero.

We assume that the shipper must satisfy an exogenous demand $X_{t}$, i.i.d. for each integer value of time $t$, that is a stationary process whose probability distribution is at least a twice differentiable unimodal distribution $F_{X}(X)$ on a support $\left[0, Q_{H i}\right]$ with $0<Q_{H i}$ and density $f_{X}(X)$, mean $\mu_{X}, 0<\mu_{X}<Q_{H i}$ and variance $\sigma_{X}^{2}$. The spot market price of transport capacity $P$ is also assumed to be an exogenous variable with similar characteristics $\left(P, F_{P}(p), f_{P}(p), \mu_{P}, \sigma_{P}^{2}\right)$ and taking values in the interval $\left[v, P_{H i}\right]$ with $0<v<P_{H i}, v$ being the variable marginal transport cost common to all carriers and $P_{H i}$ being a large value compared to $\mu_{P}$. In the same way, let us call $F$ the continuous, twice-differentiable joint unimodal distribution and $f$ the joint density function of $P$ and $X$ with mean $\mu$, variance $\sigma^{2}$ and correlation coefficient $\rho$, $(0 \leq \rho \leq 1) .{ }^{4}$ Demand and spot market are usually positively correlated because when all shippers in an industry face increased demand, they will make excess capacity scarce by their joint calls on transport capacity, thus pushing up its price for any yet available. There can exist realisations of high demand and low spot prices as well as low demand with high spot prices. ${ }^{5}$ However, we shall also present a case where spot market price is fixed and demand is a random variable.

Our model is of wider application than the one exposed in Seifert et al. (2004) because in that paper the market spot price and demand are modelled as a bivariate normal distribution only. In the present model, any bivariate unimodal distribution can be plugged in.

\subsection{Prior mutual selection of shipper and carrier and cost thereof}

The shipper and the carrier have selected each other either through previous transport experience or because the carrier has been selected through an active search among potential carriers by the shipper. The carrier has also been active in soliciting the business of the shipper. This type of relationship is known in the marketing literature as 'relationship marketing' as presented in Knemeyer et al. (2003) and references therein. According to the field study in Knemeyer et al. (2003), logistics outsourcing involves investments in specific assets and non-retrievable commitments of resources by both partners. These pre-existing costs are an incentive to play for both actors. Even though this investment is sunk when the first stage begins, each party knows that a breach of trust represented by a failure to honour the contract will trigger immediate rupture of relations. The game stops at this point and none can start between those players in the future (reputation effect). The cost is not only the foregone revenue but the necessity for each to look for another partner and hence incur the information investment again. 
As recognised in Reichheld and Sasser (1993), the process of losing clients and finding new ones (client churn rate) is common but costly. The trigger strategy for the shipper of refusing to transact in the future with this carrier will be considered a credible threat.

\subsection{Performance record and trust}

After each game, both know of the performance history of the other so that trust and records become important factors. Both players make a tally of the performance of the other player and the induced cost to him of the eventual shortfall in performance of the other. This tally of past costs is extrapolated to the remaining games to be played. If this probable estimated cost is higher than the remaining amortisation cost to him of the relation specific investment, he is better off by terminating the relationship and start a new one with another player. Thus, the higher the initial relation specific investments, the higher the lock-in effect.

Another effect is that if one player must invest much more than the other, he will feel much more bound by this lock-in effect. This difference translates into a difference in performance: the player who invests less has the least incentive to deliver the expected performance.

\section{$3.5 \quad$ games of two-stage decision processes}

There are $N$ games to be played if no contract is ever broken, $N$ being a finite number representing the long term decision planning horizon. At the end of the $N$ games, both players recover their 'liberty' to trade with the same or other players in the sense that the sunk information and managerial costs have come to the end of their useful economic lives and hence new investments have to be made to create a link to a 'new' player for a 'new' set of $N$ games. To satisfy demand addressed to the shipper, the players face a two-stage stochastic decision process within each game. In the first stage, they have to set up a transport arrangement whereby the shipper gets a privileged access to a certain transport capacity and the carrier ensures use of a certain amount of his overall capacity. In the second stage, the two actors operate according to this arrangement given the realisations of demand and spot market price. At the end of the second stage, payout occurs and the game is finished.

Let us now look in detail at how the first stage works.

To define the contract which shall be retained, both players engage in iterative alternating-offers bargaining as presented in Rubinstein (1982). We concur with Lariviere (1999) and reference to Salanié (1997) in that properly modelling bargaining is sufficiently complex that no consensus exists regarding the appropriate equilibrium concept.

If both select the same contract and agree on the contract terms, then the first stage is over, the second can then start (see Figure 1).

The second stage extends over $n$ periods. In each period from $t_{1}$ to $t_{n}$ (say every day), i.i.d. demands and spot prices are revealed and the shipper buys sufficient transport capacity from the carrier to meet the demand addressed to her. In each period, if the revealed demand exceeds committed capacity, both shipper and carrier refer $t$ the going spot market price at which this remaining demand is to be carried. Delivery and payout occurs in the last period, the game stops, each player records the performance of the other, eventual penalties are paid out, and a new game can start. 
Figure $1 N$ games with $n+1$ smaller periods in each

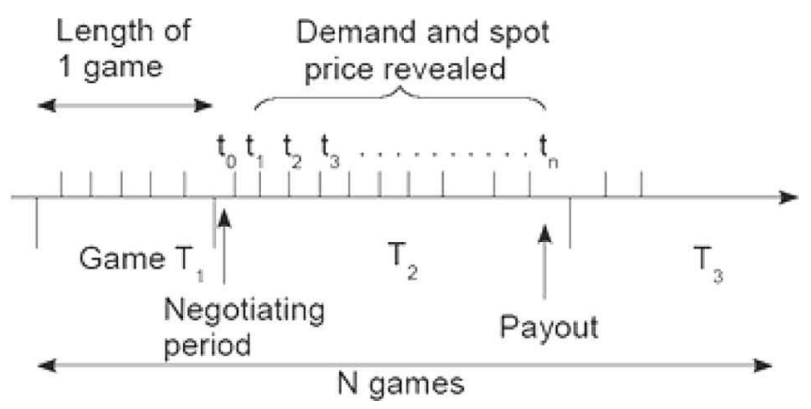

We propose here to restrict our comparison to a closed choice of mechanisms, with no possibility to re-open a contract once it has been signed and with contracts that are not contingent on one another. So each player has the choice among three contracts and not signing a contract. The outcome of the choice is a three column and three row matrix where the interesting cells are the ones on the diagonal: only if both choose the same contract and agree on the specific parameters is this stage finished. The remaining cells are the cases where the players do not agree.

If a contract and attending parameters are chosen, this stage is finished, the contract is signed and both start to operate within it: the second stage begins.

In all other cases, both players have to make a new offer represented by a type of contract and corresponding contract parameters. If a player cannot be satisfied by any offer the other player submits, he can decide to refuse to enter in a contract and this particular game is over and the second stage never starts. In the case that both choose not to sign a contract, the first and second stage are considered over, a new game can start. This possibility represents the reservation profit level given outside opportunities. The carrier can allocate more transport capacity to some other customer (Corbett et al., 2004). In the same way, the shipper can decide to privilege another carrier within her pool of known carriers. In the following, we normalise the participation and individual rationality constraints for both at zero. Intertemporal relationship is still guaranteed by the mutual initial investments by each player in 'goodwill' which still has to be amortised and the performance and demand records that each keeps.

\subsection{Fixed and variable cost of the carrier}

A convex cost function could have been modelled with different parameters for both fixed and variable costs (respectively $K$ and $c$ ) for own or third-party capacity, but this adds significantly to the calculations without adding value to the demonstration. We consider here that the required capacity allocation, result of the strategic decision of the carrier to work with this shipper, takes into account the signals which the latter sends to the former regarding the forecast demand that will have to be transported. Instead, all costs have been considered to be agglomerated into a fixed cost $K$ and a variable cost $c$, given a fixed capacity set ex ante.

Spinler and Huchzermeier (2006) distinguishes between the marginal cost of production related to long-term contract-based capacity allocation and the marginal cost of production associated with short-term spot allocation. We assume, along with Spinler and Huchzermeier, that the marginal cost is lower for the contract than 
for the spot market. In Spinler and Huchzermeier (2006), both costs are assumed to increase in the state of the economy, a modelling artefact which has been eliminated by considering the marginal cost and the spot market to be independent. The carrier is an active participant in the spot market in his own right as he daily has to balance his transport requirements and available capacity. As they are independent of the transport requirement expressed by the shipper, the information cost and all other costs incurred while dealing in the spot market are considered as included in his fixed cost.

\subsection{Carrier's estimation of shipper's demand}

The carrier does not know the demand distribution of the shipper and therefore must make an educated guess. Before the start of the first game, this guess is tantamount to estimating the probable demands based on the carrier's knowledge of the industry the shipper is in. The carrier is embedded in a Bayesian universe in which parties have an a priori belief on the information they do not possess, and they revise this belief as the interaction unfolds. So, the carrier has a Bayesian belief about the distribution of the expected demand to be carried $q_{0}$ in an interval $\left[q_{0}, \overline{q_{0}}\right]$. The cumulative distribution function is $F_{X}(q)$ (with $F_{X}\left(\underline{q_{0}}\right)=0$ and $F_{X}\left(\overline{q_{0}}\right)=1$ ) and $f_{X}\left(q_{0}\right)$ as the continuous and strictly positive density on $\left[\underline{q_{0}}, \overline{q_{0}}\right]$.

Before the first game, the carrier will exaggerate the possible cost by underestimating the average demand and overestimating the variance of this demand, inflating both variable and fixed cost. Game after game, he is able to update his estimates of demand and corresponding distribution function and refines his evaluation of actual variable and fixed costs. ${ }^{6}$

The carrier wishes to know what to expect from the shipper in terms of capacity. To that end, he builds a menu of prices in one contract which enables him to deduct from the answer of the shipper the expected capacity which will be required. This problem was first mooted in Myerson (1979), well presented and treated in Laffont and Tirole (1993) and extended to mechanism design with direct revelation and non-linear utility functions in finite-space in Lovejoy (2006). The problem here is of this same type. We argue that since this is a repeated game between the same actors, the carrier will have be able to update the expected demand game after game in such a way that it is in the shipper's interest to indicate even in the first game her expected level of demand. We conjecture that this principal agent problem can be solved by devising a menu of fixed fees and capacity commitments which discriminates among shippers by solving their incentive compatibility constraints.

\subsection{Shipper costs and estimates}

Before the start of a game, the shipper observes the demand distribution parameter $\mu_{X}$ which defines $F_{X}(x)$, and which we interpret as her forecast of demand. From her own past experience and polls from other carriers, she derives a budget $B$ for transport which covers all periods of a contract. Ex post, it may be that the overall transport cost (including the cost of procuring some transport capacity from the spot market) is higher than $B$. This extra cost is still paid to the carrier but internally such an event is considered as a smudge on the shipper's ability to budget correctly. 


\subsection{Risk aversion}

Risk aversion is reduced to the possible lack of transport capacity when needed by the shipper and by the possible absence of cargo to load by the carrier. This motivates the contract in the first place but does not constrain the utility functions of the players within each game (once a contract has been signed). This feature is not how Seifert et al. (2004) has chosen to represent the risk aversion of the buyer. There, risk aversion is represented by including in her objective function the variance of profits times a factor $k$ : as $k$ increases, the more risk-averse is the buyer. ${ }^{7}$

The Table 1 lists all the notation in this chapter.

\subsection{Types of contracts}

The three contracts chosen here, as mentioned in the literature review, represent individually optimal contracts to coordinate a shipper and carrier when information is asymmetric. Here, in a key difference from other models, the process of buying transport capacity from the spot market is assumed to entail a higher cost than the one attributed to contract buying. This is to represent that information gathering, service quality and price discovery all cost significantly more than the transaction cost involved in buying from the contracted carrier. As we shall see lower, this helps to steer away from trivial situations where the shipper might always be better off buying capacity from the spot market or pushing for a watered down PRC.

The objective maximising functions are described for the shipper and carrier under three settings: PRC, a MPC and a contract with single price but with QFC.

We now proceed to describe the contract mechanisms.

\subsection{Price-only Relational Contract}

In this form of contract, the shipper's cost function is:

$$
V_{1}\left(x_{t}, p_{r}\right)=p_{r} x_{t}, \quad \forall t \in\{1, \ldots, n\}
$$

where $p_{r}$ is the negotiated price ex-ante and $x_{t}$ is the realised demand in period $t$. The carrier's objective function is the profit function

$$
\pi_{1}\left(x_{t}, p_{r}\right)=p_{r} x_{t}-c x_{t}-K, \quad \forall t \in\{1, \ldots, n\} .
$$

In this type of contract, the shipper does not make any demand forecast and the carrier cannot make an educated decision on the capacity to allocate to the shipper's requirements. The downfall is that the shipper cannot be sure that the carrier will always satisfy all her transport capacity requirements.

It must be noted that in this type of contract, if the shipper's cost of accessing the spot market $I=0$, the price which makes the contract eligible would be $p_{r}=\mu_{P}$, the mean spot market price, if the specific assets that the shipper has invested in her relationship to this carrier were fully amortised. Our model specifically excludes this case since we consider that the negotiation takes place within the number $N$ of games, the required time to amortise the specific assets that both the carrier and the shipper have invested in. 
Table 1 Table of notations

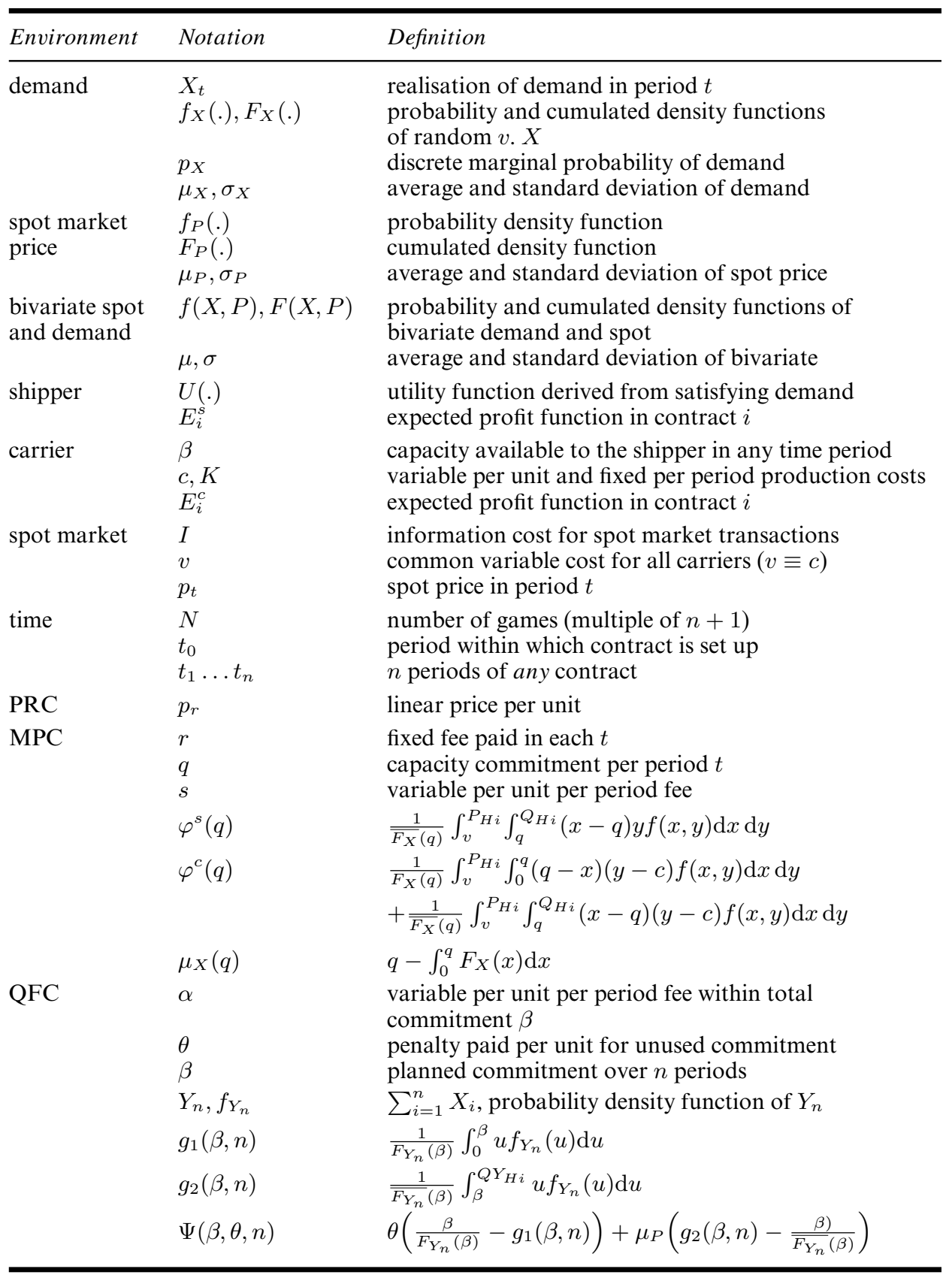

In the general case, we spell the expected residual budget or profit at both firms as

$$
\begin{aligned}
E_{1}^{s}\left(p_{r}\right) & =B-\mathbb{E}\left(\sum_{t=1}^{n} V_{1}\left(x_{t}, p_{r}\right)\right) \\
& =B-n p_{r} \mu_{X} ;
\end{aligned}
$$




$$
\begin{aligned}
E_{1}^{c}\left(p_{r}\right) & =\mathbb{E}\left(\sum_{t=1}^{n} \pi_{1}\left(x_{t}, p_{r}\right)\right) \\
& =n\left(p_{r} \mu_{X}-c \mu_{X}-K\right) ;
\end{aligned}
$$

with superscript $c$ for the carrier, $s$ for the shipper and $\mathbb{E}$ the expectation sign.

Some mention must be made here as to the ex ante choices available to the shipper and carrier. If the shipper enjoys a cost of information about available capacity in the spot market equal to $0(I=0)$, then her choice $a b$ initio is to take her transport requirements to the spot market or to the carrier, which if she hadn't incurred any costs in 'finding' the carrier in the first place, would mean that the cost $p_{r}$ of the PRC would be reduced to the mean of the spot market price $\mu_{P}$ :

$$
p_{r}=\frac{1}{\mu_{X}} \iint X P f(X, P) d X d P
$$

In fact, it is a better representation of reality to model a positive cost of information and a positive search and specific asset investment amortisation cost that the shipper incurs in procuring her transport capacity requirements. In the following, we shall consider that both these costs are not null for all transactions involving a contract: PRC, MPC or QFC.

By the individual rationality and participation constraints, we must have jointly

$$
\begin{aligned}
& B-n p_{r} \mu_{X} \geq 0 \\
& n\left(p_{r} \mu_{X}-c \mu_{X}-K\right) \geq 0 .
\end{aligned}
$$

The following condition follows directly from the above.

\section{Condition 1: Conditions for a PRC}

For the PRC to be eligible by both supplier and buyer, the chosen $p_{r}$ must meet the following conditions:

$$
c+\frac{K}{\mu_{X}} \leq p_{r} \leq \frac{B}{n \mu_{X}} .
$$

Save for the trivial case where the supplier's costs are higher than the buyer's budget, the above proposition admits a set of values apt to satisfy both players.

\subsection{Minimum Purchase Commitment contract}

The MPC consists in a fixed fee $r$ that the shipper agrees to pay the carrier at each of the $n$ periods within the contract, a capacity commitment $q$ and a variable fee $s$ for each unit effectively carried in the period. ${ }^{8}$ The fixed fee is paid whether the shipper takes delivery of the committed transport capacity or not. This type of contract plays two roles: convincing the carrier that her future requirements are genuine and inducing him to reserve adequate capacity so that he will not fail her.

This is very similar to the supply chain management model presented in Cachon and Lariviere (2001) for the price-only contract, voluntary compliance, asymmetric 
information case. As in Cachon and Lariviere's case, our model also falls into the type of economic models known as 'signalling' models. In it, Cachon and Lariviere present a manufacturer who requires from a supplier $K \geq 0$ units of production capacity at $w(K)$ wholesale price, proportionate to the capacity required. The point is to motivate the supplier to build or reserve adequate capacity so as to be able to serve the realised demand. The manufacturer, being private to the forecast demand, wishes to communicate this forecast credibly. He does so by paying an additional 'lump sum' to the supplier ex ante. Cachon and Lariviere establish in their Lemma 2 that if the manufacturer can make a request for a capacity somewhat larger than what he expects and commit to a lump sum payment ex ante, then the expected demand is credibly signalled to the supplier. They further establish in their Theorem 6 that the manufacturer's profit is higher when he requests more capacity than what he expects to buy when demand is realised. We use these results in the following as the settings are clearly similar. One notable difference however is that the wholesale price paid by the manufacturer in their model requires him to know the production costs of the supplier, an information which the shipper does not possess in our model and which is frequently unavailable in the transport industry.

The fact that the shipper also buys transport capacity from the spot market is also envisaged in Cachon and Lariviere (2001) in Section 6: 'a second source' where it is showed that the single supplier model can be used to study a two supplier model and still derive the same conclusions. The information cost $I$ of our model can be included as part of the extra cost of the second supplier in their model.

Hence, we consider that the shipper will signal her expected demand per period through her proposed parameters for a MPC. Our discussion of the evaluation of the variable per unit fee $s$ shall depart from their model.

From period $t_{1}$ to period $t_{n}$ demand and the spot market price are realised. The shipper buys the necessary transport capacity from the carrier who delivers demands at or below $q$ at the contracted price and extra demand above $q$ at the spot market price. The remainder of committed capacity is sold on the spot market. Payout occurs at every period. The game ends after period $n$.

For a given outcome the shipper's cost function is per period

$$
V_{2}\left(x_{t}, p_{t}\right)= \begin{cases}r+q s+\left(x_{t}-q\right) p_{t}+I, & \text { when } x_{t}>q, \\ r+x_{t} s, & \text { when } x_{t} \leq q\end{cases}
$$

whereas, for the carrier it is

$$
\pi_{2}\left(x_{t}\right)= \begin{cases}r+q(s-c)+(x-q)\left(p_{t}-c\right)-K, & \text { when } x_{t}>q, \\ r+x_{t}(s-c)+\left(q-x_{t}\right)\left(p_{t}-c\right)-K, & \text { when } x_{t} \leq q\end{cases}
$$

The expected profit for shipper and carrier are functions of $q, s, r$, contract parameters which become decision variables of both players. From the definition of the conditional distribution and of conditional expected values, we define new functions $\varphi$ such that

$$
\begin{aligned}
\varphi^{s}(q) & =\mathbb{E}\left(\left(x_{t}-q\right) p_{t} \mid X>q\right), \quad \forall t \in\{1, \ldots, n\} \\
& =\frac{1}{\overline{F_{X}}(q)} \int_{v}^{P_{H i}} \int_{q}^{Q_{H i}}(x-q) y f(x, y) \mathrm{d} x \mathrm{~d} y
\end{aligned}
$$




$$
\begin{aligned}
\varphi^{c}(q)= & \mathbb{E}\left(\left(q-x_{t}\right)\left(p_{t}-c\right) \mid X \leq q\right)+\mathbb{E}\left((x-q)\left(p_{t}-c\right) \mid X>q\right), \\
& \forall t \in\{1, \ldots, n\} \\
= & \frac{1}{F_{X}(q)} \int_{v}^{P_{H i}} \int_{0}^{q}(q-x)(y-c) f(x, y) \mathrm{d} x \mathrm{~d} y \\
& +\frac{1}{\overline{F_{X}}(q)} \int_{v}^{P_{H i}} \int_{q}^{Q_{H i}}(x-q)(y-c) f(x, y) \mathrm{d} x \mathrm{~d} y,
\end{aligned}
$$

with $\overline{F_{X}}(q)=1-F_{X}(q)$.

The carrier fills the transport requirement of the shipper subject to the limit of commitment $q$ made by the shipper. Let $\mu_{X}(q)$ be expected transport under constraint of the committed capacity $q^{9}$ :

$$
\begin{aligned}
\mu_{X}(q) & =\mathbb{E}\left[X-(X-q)^{+}\right] \\
& =q-\int_{0}^{q} F_{X}(x) d x .
\end{aligned}
$$

So in each game where this contract has been chosen the buyer's expected profit is

$$
\begin{aligned}
E_{2}^{s}(q, s, r) & =B-\mathbb{E}\left(\sum_{t=1}^{n} V_{2}\left(x_{t}, p_{t}\right)\right) \\
& =B+n\left[-r-s \mu_{X}(q)-I \overline{F_{X}}(q)-\varphi^{s}(q)\right] .
\end{aligned}
$$

For the supplier:

$$
\begin{aligned}
E_{2}^{c}(q, s, r) & =\mathbb{E}\left(\pi_{2}(X, P)\right) \\
& =n\left(r+(s-c) \mu_{X}(q)+\varphi^{c}(q)-K\right) .
\end{aligned}
$$

For each to choose this contract, the individual rationality constraints are:

$$
\left\{\begin{array}{l}
B / n-r-s \mu_{X}(q)-I \overline{F_{X}}(q)-\varphi^{s}(q) \geq 0 \\
r+(s-c) \mu_{X}(q)-K+\varphi^{c}(q) \geq 0
\end{array}\right.
$$

On the domains of $q$ and $s$ in which the shipper's objective functions is concave, the interesting variable to determine is the optimal quantity $q^{*}$, object of the commitment.

Theorem 2: In the case of a Minimum Purchase Commitment (MPC), if and only if the shipper's objective function is concave, the optimal parameters $q$ and $s$ which satisfy the shipper are solution to the equation

$$
s=\frac{I f_{X}(q)-\varphi^{s \prime}(q)}{\overline{F_{X}}(q)}
$$

see Appendix A for the proof.

The carrier will always be looking for the highest possible commitment within his other constraints. This result can be compared to the one obtained in Spinler and Huchzermeier (2006), where the boundaries over optimal parameters of the options to 
be bought are expressed in terms of one another in Corollary 3 and are also only optimal to the buyer. Further, in Spinler and Huchzermeier (2006), the result is only valid when a mapping of the demand to the spot price exists whereas here, any relationship between demand and spot price is accommodated.

For the MPC to be retained, the individual rationality constraints have to be satisfied in every period. Given that the shipper has tailored $B$ to be sufficiently large as to accommodate demand and possible values of $q, r$ and $s$ (from past experience and observation of other similar arrangements in the market), and given that the carrier will not wish to contemplate any offer from the shipper which does not allow him to cover his fixed cost $K$, the mutually agreeable sets of parameters $\{q, r, s\}$ is non-empty. In the case of a concave objective function for the shipper, this leads us to spell out the following condition:

\section{Condition 3: Conditions for a MPC}

For the MPC contract to be chosen by both supplier and buyer and also enjoy the coordinating powers described in Cachon and Lariviere (2001), ${ }^{10}$ the contract parameters $q, r$ and s must meet the following conditions

$$
\left\{\begin{array}{l}
r \leq \frac{B}{n}-s \mu_{X}(q)-I \overline{F_{X}}(q)-\varphi^{s}(q) \\
r \geq K-\varphi^{c}(q)-(s-c) \mu_{X}(q) \\
s=\frac{1}{\overline{F_{X}}(q)}\left(I f_{X}(q)-\varphi^{s \prime}(q)\right) \\
r, s \geq 0, \quad q>0
\end{array}\right.
$$

This result can be related to the result from the use of the 'advanced contract' and asymmetric information in the model presented in Cachon and Lariviere (2001), from which the model is inspired.

One difference is that in our case $q$ is the total capacity reserved by the carrier for the shipper's needs, whereas Cachon and Lariviere make a distinction between the 'firm commitment' by the supplier to the manufacturer (analogous to the commitment by the carrier to the shipper) and the total capacity that the supplier actually reserves to the manufacturer (shipper, in our case). However, they make no attempt at characterising literally the optimal capacity in the asymmetric information, voluntary compliance case, which is what we attempt to do here.

Another difference is that they consider that the demand not met by the contract is lost to the manufacturer, whereas we let the shipper solve extra demands she may receive using the spot market price.

\subsection{Quantity Flexibility Contract}

Consider the case of a price $\alpha$ per unit of cargo and a minimum quantity commitment $\beta$ by the shipper to the carrier over $n$ periods. To ensure coordination, at the end of the game, a penalty ${ }^{11}$ per unit $\theta$ is charged by the carrier to the shipper for all transport requirements over the $n$ periods which the shipper did not need. That is, the total transported units are summed and compared to $\beta$, the amount paid by the shipper to the carrier equals the shortfall in units times the penalty. 
If the shipper has had $\beta$ carried before the end of the contract, she can purchase additional capacities from the carrier at the revealed going mean spot market price $P_{i}$ for the remaining periods within the total $n$. This is possible by making an adjustment at the end of the $n$ periods to the observed daily spot market price. Since she knows the carrier, she does not incur the information cost $I$. The carrier will be at the receiving end of the variance of the demand that the shipper faces. He will accommodate within his own transport capacity what he can and derive the excess to the spot market in pursuance of his best interest: satisfy his customer, increase his knowledge of the demands that the shipper faces for future reference in future bargaining rounds in the next games and finally to increase his presence and visibility in the spot market. We argue that the payout he receives from such dealings will not generate any profit but the other intangible side benefits should still make it worthwhile.

Payout and penalty occur at the end of the $n$ periods. Note that in this contract the penalty, if due, is paid only after revelation of the demand of the nth period.

The shipper's and carrier's expected profit functions can now be written using the random variable of the sums of demands:

$$
\begin{aligned}
& E_{3}^{s}(\alpha, \beta, \theta, n)=B-n \alpha \mu_{X}-\Psi(\beta, \theta, n) \\
& E_{3}^{c}(\alpha, \beta, \theta, n)=n\left[\alpha \mu_{X}-c \mu_{X}-K\right]+\Psi(\beta, \theta, n),
\end{aligned}
$$

with $\Psi$ defined as

$$
\Psi(\beta, \theta, n)=\theta\left(\frac{\beta}{F_{Y_{n}}(\beta)}-g_{1}(\beta, n)\right)+\mu_{P}\left(g_{2}(\beta, n)-\frac{\beta}{\overline{F_{Y_{n}}}(\beta)}\right) .
$$

Let us define $g_{1}(.,$.$) the conditional or truncated mean of the sum of n$ demands being less than $\beta$ and $g_{2}(.,$.$) the conditional mean of the sum of n$ demands being higher than $\beta$ :

$$
g_{1}(\beta, n)=\frac{1}{F_{Y_{n}}(\beta)} \int_{0}^{\beta} u f_{Y_{n}}(u) \mathrm{d} u, \quad g_{2}(\beta, n)=\frac{1}{\overline{F_{Y_{n}}}(\beta)} \int_{\beta}^{Q Y_{H i}} u f_{Y_{n}}(u) \mathrm{d} u
$$

where $Q_{H i}$ and $P_{H i}$ are suitably high numbers above which the probability of a demand or a spot market price are considered suitably low. ${ }^{12}$

The definitions of the functions $g_{1}$ and $g_{2}$ are also in Table 1 .

Proof in Appendix B.

The conditions and the characterisation of the optimal values of $\alpha, \beta, \theta$ and $n$ are discussed in Appendix C.

\section{Condition 4: Conditions for existence of a QFC}

For the QFC to be chosen by both shipper and carrier, the contract parameters $\alpha, \beta, \theta, n$ must meet the following conditions

$$
\left\{\begin{array}{l}
\alpha \geq 0, \wedge \theta \geq 0, \wedge \beta>0 \\
\theta\left(\frac{\beta}{F_{Y_{n}}(\beta)}-g_{1}(\beta, n)\right) \leq B(n)-n \alpha \mu_{X}-\mu_{P}\left(g_{2}(\beta, n)-\frac{\beta}{\overline{F_{Y_{n}}}(\beta)}\right) \\
\theta\left(\frac{\beta}{F_{Y_{n}}(\beta)}-g_{1}(\beta, n)\right) \geq n K+n c \mu_{X}-n \alpha \mu_{X}-\mu_{P}\left(g_{2}(\beta, n)-\frac{\beta}{\overline{F_{Y_{n}}}(\beta)}\right) .
\end{array}\right.
$$




\section{Choosing a contract}

Which contract should the players choose? The model assumes that the carrier is unaware of the forecast of demand made by the shipper. He relies on the past demands he has observed in previous games. In our particular case the Theorem 7 in Cachon and Lariviere (2001) prove that lump sum payments in these type of settings generate separating equilibria so that the carrier can use the proposed contracts and corresponding parameters as indications of the shipper's demand forecast. In our model, we have two contracts which induce lump sum payments: $r$ in the MPC and $\theta$, the penalty, in the QFC. So any offer by the shipper in any of those contracts signals the level of forecasted demand.

We first compare each contract to one another, we then spell the conditions for the dominating contract.

\subsection{Minimum commitment vs. Price-only Relational Contract}

The difference between the expected values to the buyer of the MPC and relational contract is labelled $D_{2-1}$; a function of the contract parameters $p_{r}, q, s$ and $r$. In the following, the decision variables shall be omitted when no confusion can ensue to alleviate the notation.

From (3) and (11) for the shipper and from (4) and (12) for the carrier,

$$
\begin{aligned}
D_{2-1}^{s} & =E_{2}^{s}(q, s, r)-E_{1}^{s}\left(p_{r}\right) \\
& =n\left[-r-s \mu_{X}(q)-I\left(1-F_{X}(q)\right)-\varphi^{s}(q)+p_{r} \mu_{X}\right] \\
D_{2-1}^{c} & =E_{2}^{c}(q, s, r)-E_{1}^{c}\left(p_{r}\right) \\
& =n\left[r+s \mu_{X}(q)+\varphi^{c}(q)-p_{r} \mu_{X}-c\left(\mu_{X}(q)-\mu_{X}\right)\right] .
\end{aligned}
$$

We are interested in the signs of the differences: for both to choose the same contract, the differences must be of the same sign.

$$
\begin{aligned}
& D_{2-1}^{c} \geq 0 \wedge D_{2-1}^{s} \geq 0 \Rightarrow \text { MPC weakly preferred } \\
& D_{2-1}^{c}<0 \wedge D_{2-1}^{s}<0 \Rightarrow \text { PRC strictly preferred. }
\end{aligned}
$$

However, the conditions on $q$ from Condition 3 must also be met for a MPC to prevail. All of which lead to the following set of inequalities.

\section{Condition 5: Conditions of preeminence of a MPC over a PRC}

The MPC will be weakly preferred over the PRC when Condition 3 and

$$
\left\{\begin{array}{l}
r \leq p_{r} \mu_{X}-I \overline{F_{X}}(q)-\varphi^{s}(q)-s \mu_{X}(q) \\
r \geq p_{r} \mu_{X}-\varphi^{c}(q)+c\left(\mu_{X}(q)-\mu_{X}\right)-s \mu_{X}(q),
\end{array}\right.
$$

are fulfilled. The PRC will be preferred when

$$
\left\{\begin{array}{l}
r>p_{r} \mu_{X}-I \overline{F_{X}}(q)-\varphi^{s}(q)-s \mu_{X}(q) \\
r<p_{r} \mu_{X}-\varphi^{c}(q)+c\left(\mu_{X}(q)-\mu_{X}\right)-s \mu_{X}(q),
\end{array}\right.
$$

and Condition 1 are satisfied. 


\subsection{Quantity flexibility vs. Price-only Relational Contract}

Let $D_{3-1}$ be the function of the difference between QFC and PRC over $n$ periods. We are again interested in the sign of this function. The difference $D_{3-1}$ is a function of the decision variables $p_{r}, \alpha, \beta, \theta$, and $n$.

From (3), (4) and (16)

$$
\begin{aligned}
& D_{3-1}^{s}=n\left[p_{r} \mu_{X}-\alpha \mu_{X}\right]-\Psi(\beta, \theta, n), \\
& D_{3-1}^{c}=n\left[\alpha \mu_{X}-p_{r} \mu_{X}\right]+\Psi(\beta, \theta, n),
\end{aligned}
$$

which are exactly opposite from each other. In practical terms, for both players to choose the QFC contract means that both (24) and (25) must be positive or null at the same time.

The only solution is when both contracts yield the same utility to both players. Hence,

$$
p_{r} \mu_{X}=\alpha \mu_{X}+\frac{\Psi(\beta, \theta, n)}{n},
$$

because $n>0$. For all other values, each player would not choose the same contract. The other conditions which render the QFC or the PRC eligible also have to be satisfied. The following set of conditions is enunciated.

\section{Condition 6: Conditions for a QFC to be equivalent to a PRC}

Both carrier and shipper are indifferent between PRC and QFC if

$$
p_{r}=\alpha+\frac{\Psi(\beta, \theta, n)}{n \mu_{X}}
$$

and Conditions 1 and 4 are satisfied. In all other cases, no agreement on these two contracts can be reached.

\subsection{Quantity flexibility vs. minimum commitment}

Building from previous results, we now try to help the shipper and carrier choose between QFC and MPC, but also include the conditions for both contracts to dominate the PRC. Let us call $D_{3-2}$ the functions of the differences between QFC and MPC in terms of the decision variables $\alpha, \beta, \theta, n, r, s$ and $q$. According to (11), (12) and (16),

$$
\begin{aligned}
& D_{3-2}^{s}=n\left[-\alpha \mu_{X}+r+s \mu_{X}(q)+I \overline{F_{X}}(q)+\varphi^{s}(q)\right]-\Psi(\beta, \theta, n), \\
& D_{3-2}^{c}=n\left[\mu_{X}(\alpha-c)-r-(s-c) \mu_{X}(q)-\varphi^{c}(q)+\Psi(\beta, \theta, n)\right] .
\end{aligned}
$$

The following set of conditions can be enunciated (Proof in Annex D).

\section{Condition 7: Conditions of dominance of a QFC over a MPC}

When carrier and shipper have the choice between a QFC and a MPC contract, they will choose the QFC when Conditions 3 and 4 and the following Condition is satisfied

$$
\left\{\begin{array}{l}
r+s \mu_{X}(q)+I \overline{F_{X}}(q)+\varphi^{s}(q)>\Psi(\beta, \theta, n) / n+\mu_{X} \alpha \\
c \mu_{X}+r+(s-c) \mu_{X}(q)+\varphi^{c}(q)<\Psi(\beta, \theta, n) / n+\mu_{X} \alpha .
\end{array}\right.
$$


The MPC shall prevail when Conditions 3, 4 and

$$
\left\{\begin{array}{l}
r+s \mu_{X}(q)+I \overline{F_{X}}(q)+\varphi^{s}(q)<\Psi(\beta, \theta, n) / n+\mu_{X} \alpha \\
c \mu_{X}+r+(s-c) \mu_{X}(q)+\varphi^{c}(q)>\Psi(\beta, \theta, n) / n+\mu_{X} \alpha
\end{array}\right.
$$

are satisfied. Both will be equivalent when Conditions 3, 4 and

$$
\left\{\begin{array}{l}
r+s \mu_{X}(q)+I \overline{F_{X}}(q)+\varphi^{s}(q)=\Psi(\beta, \theta, n) / n+\mu_{X} \alpha \\
c \mu_{X}+r+(s-c) \mu_{X}(q)+\varphi^{c}(q)=\Psi(\beta, \theta, n) / n+\mu_{X} \alpha
\end{array}\right.
$$

are satisfied.

\subsection{Conditions for a contract to dominate the others}

We now consider that both carrier and shipper have the choice among all three contracts at the same time. For this to happen, all three have to be already agreeable per se and also be mutually compatible. This means that we must have simultaneously the choice of a PRC agreeable to both and the choice of a QFC agreeable to both, so that we require Condition 6 to be satisfied before even comparing them to the MPC. In this case, we have Conditions 5 and 7 which become equivalent. To recapitulate, the conditions for each contract to dominate are the following.

\section{Condition 8: Conditions for dominance of a PRC over a MPC and equivalence with} a QFC

A PRC will be equivalent to a QFC and preferred over a MPC when

$$
\left\{\begin{array}{l}
p_{r}=\alpha+\frac{\Psi(\beta, \theta, n)}{n \mu_{X}} \\
c+\frac{K}{\mu_{X}}<p_{r} \leq \frac{B}{n \mu_{X}} \\
r>p_{r} \mu_{X}-I \overline{F_{X}}(q)-\varphi^{s}(q)-s \mu_{X}(q) \\
r<p_{r} \mu_{X}-\varphi^{c}(q)+c\left(\mu_{X}(q)-\mu_{X}\right)-s \mu_{X}(q) .
\end{array}\right.
$$

\section{Condition 9: Conditions for dominance of a MPC over both a QFC and a PRC}

A MPC will be preferred when

$$
\left\{\begin{array}{l}
r \leq \frac{B}{n}-s \mu_{X}(q)-I \overline{F_{X}}(q)-\varphi^{s}(q) \\
r \geq K-\varphi^{c}(q)-(s-c) \mu_{X}(q) \\
r<p_{r} \mu_{X}-I \overline{F_{X}}(q)-\varphi^{s}(q)-s \mu_{X}(q) \\
r>p_{r} \mu_{X}-\varphi^{c}(q)+c\left(\mu_{X}(q)-\mu_{X}\right)-s \mu_{X}(q) .
\end{array}\right.
$$




\section{Condition 10: Conditions for a QFC to dominate the MPC and be equivalent to the PRC}

The QFC will be preferred over the MPC and weakly preferred over the PRC when the following conditions are met

$$
\left\{\begin{array}{l}
\alpha \geq 0, \wedge \theta \geq 0, \wedge \beta>0, \wedge n>10 \\
p_{r}=\alpha+\frac{\Psi(\beta, \theta, n)}{n \mu_{X}} \\
\theta \leq \frac{1}{\frac{\beta}{F_{Y_{n}}(\beta)}-g_{1}(\beta, n)}\left[B(n)-n \alpha \mu_{X}-\mu_{P}\left(g_{2}(\beta, n)-\frac{\beta}{\overline{F_{Y_{n}}}(q)}\right)\right] \\
\theta \geq \frac{\beta}{\frac{\beta}{F_{Y_{n}}(\beta)}-g_{1}(\beta, n)}\left[n K+n c \mu_{X}-n \alpha \mu_{X}-\mu_{P}\left(g_{2}(\beta, n)-\frac{\beta}{\overline{F_{Y_{n}}}(q)}\right)\right] . \\
r+s \mu_{X}(q)+I \overline{F_{X}}(q)+\varphi^{s}(q)>p_{r} \mu_{X} \\
c \mu_{X}+r+(s-c) \mu_{X}(q)+\varphi^{c}(q)<p_{r} \mu_{X}
\end{array}\right.
$$

Evidently these are not trivial results, they change from previous ones as stated in literature because of the endogenous choice of transaction form.

If the above sets of conditions for eligibility of a contract are not met, no agreement can be reached and this particular game ends. The carrier will prefer to attend to his other customers, whereas the shipper will look among her other carriers. This does not preclude that in the following game both agree on a common contract and corresponding parameters. ${ }^{13}$

To grasp the results and their significance, let us present three numerical examples. The first presents a case where both demand and spot market prices for transport fluctuate in a bivariate normal distribution. In the second, we fix the transport price and let demand fluctuate in a normal distribution. The last example is a case where both carrier and shipper use only the spot market and do not interact together: this last example uses also a bivariate normal distribution of demand and spot market prices. Further examples applying exponential distributions of demand and prices have not been presented here because the results are functionally the same.

\section{Numerical examples}

\subsection{First example: a binormal stochastic process}

We instantiate the preceding results in the following way. Let $f(X, P)$ be a bivariate normal distribution with their supports, the information cost for the spot market, the carrier's fixed and variable cost take the following values:

$$
\begin{aligned}
& \mu_{X}=10, \quad \sigma_{X}=3, \quad \mu_{P}=8, \quad \sigma_{P}=1.5, \quad \rho=0.5 \\
& I=2, \quad v=2, \quad P_{H i}=14, \quad Q_{H i}=25, \quad K=20, \quad c=1.8 .
\end{aligned}
$$

The transport budget is estimated in terms of the average per unit spot market price. So as to be on the safe side, the shipper adds a margin which we set here at $20 \%$. The transport budget is a function of the number of periods over which a contract runs

$$
B(n)=\mu_{P}(1.2) n \mu_{X}
$$


By the Central Limit Theorem, the sum of the expected demands over a large number of periods (more than 10 periods) behaves like a normal distribution which has the following characteristics:

$$
Y_{n}=\sum_{t=1}^{n} x_{t} \sim \mathcal{N}\left(n \mu_{X}, \sqrt{n} \sigma_{X}\right) \sim \mathcal{N}(10 n, 3 \sqrt{n}) .
$$

To show the impact of the choice of contract on each player, let us now evaluate the objective function of the shipper and carrier when the choice of contract is exogenously given and when the choice is endogenous. ${ }^{14}$ We present the results in Table 2.

Table 2 Bivariate demand: table of maximum contract outcomes for each player separately and parameter conditions for exogenous or endogenous choice of contract

\begin{tabular}{|c|c|c|c|c|}
\hline \multirow[b]{2}{*}{$n=40$} & \multicolumn{2}{|c|}{ Carrier } & \multicolumn{2}{|c|}{ Shipper } \\
\hline & Exogenous & Endogenous & Exogenous & Endogenous \\
\hline PRC & 2320 & 2320 & 2320 & 470 \\
\hline Variance & \multicolumn{2}{|c|}{$p_{r}=9.6, q=1$} & $\begin{array}{l}p_{r}=3.8 \\
0.21 \times 10^{6}\end{array}$ & $\begin{array}{l}p_{r}=8.43, q=1 \\
1.02 \times 10^{6}\end{array}$ \\
\hline MPC & $\begin{array}{l}5158.4 \\
q=22, s=8.87 \\
r=0,\end{array}$ & $\begin{array}{l}2926.1 \\
q=12, s=8.26 \\
r=0 \\
<9.6\end{array}$ & \multicolumn{2}{|c|}{$\begin{array}{c}q=8, s=5.98, r=0 \\
7.51<p_{r}<9.6\end{array}$} \\
\hline Variance & $1.21 \times 10^{6}$ & $0.88 \times 10^{6}$ & $0.87 \times 10^{6}$ & $0.87 \times 10^{6}$ \\
\hline QFC & $\begin{array}{l}2320 \\
\alpha=9.30\end{array}$ & $\begin{array}{l}1486 \\
\alpha=7.21\end{array}$ & $\begin{array}{l}2320 \\
\alpha=3.50\end{array}$ & $\begin{array}{l}1504 \\
\alpha=5.54\end{array}$ \\
\hline Variance & $1.28 \times 10^{6}=400$ & $\begin{array}{l}=0, q=1 \\
0.79 \times 10^{6}\end{array}$ & $\begin{array}{r}\beta=40 \\
0.21 \times 10^{6}\end{array}$ & $\begin{array}{c}\theta=0, q=1 \\
0.48 \times 10^{6}\end{array}$ \\
\hline Spot & 1680 & - & 470 & - \\
\hline Variance & $1.89 \times 10^{6}$ & - & $1.89 \times 10^{6}$ & - \\
\hline
\end{tabular}

We assume in the following that the number of periods of a game is 40 .

The details of the calculations first of the exogenous choice of each contract are relegated to the Sections A-C in the Appendix.

Once the required domains for the emergence of the contracts are defined, we compare the contracts. The details of the calculations can be found in $\mathrm{D}-\mathrm{G}$ in the Appendix. For all the contracts and corresponding solution, the variance of the objective function have been tabulated. The results are presented in two.

The choice of contracts once the comparison is endogenous yields a preference for the MPC for a large range of values; the PRC and QFC prevail only when the partners agree to a commitment per period of $q=1$ for the MPC, hardly an optimal setting when the expected demand faced by the shipper is at least of a required capacity of ten. For all practical purposes, this means that the shipper and carrier will settle for a MPC most of the time. If they do, the reader will note that neither partner can pull the cover to himself: each can expect to make a profit or retain some budget even in the extreme cases where one partner has all the bargaining power. 
If we suppose that the MPC is not retained, the choice is between two contracts which can yield the same economic result for both partners, however, if variance is an issue, one sees that the QFC would get the preference of both players. There are large domains of possible parameter sets which would satisfy both and split the supply chain's surplus more or less evenly.

Overall the choice of contract and the parameters on which both partners agree is counterintuitive and presents the case of the transport industry within the overall supply chain management literature as a supply chain member apart due to the particularities of the trade. The observations in Cachon and Lariviere (2001) about the frequency of the MPC in industry support this affirmation.

It is further interesting to note in Table 2 the hierarchy between the variance of the results to both the shipper and the carrier when the maximum yielding transport procurement contract is adopted. Clearly, the strategy to procure or sell transport capacity by using exclusively the spot market, which is given here as reference, not only produces less interesting results for both carrier and shipper (even though the carrier does not have to pay a fixed information cost to access it) but it induces the highest level of variance of the results. Such a strategy should only be adopted by shippers and carriers who cannot engage in other types of contractual arrangements or when both demand and spot market volatilities are very low or also if the specific assets that are needed by both for a relationship are of low value compared to the benefits of spot market transactions. This result is similar to the observations in the market and to results presented in supply chain management literature as in Kleindorfer and $\mathrm{Wu}$ (2003).

When the volatility of demand and spot market prices are higher, the volatility of the MPC can become as high as the one for the PRC, even for high values of $p_{r}$, undermining its interest for both players. In such a case, the QFC becomes even more attractive, especially if $\alpha$ is low.

What happens when the spot market price does not move? This is the case of a market for transport capacity which is not subject to sudden variations and hence where both shippers and carriers can withhold excess demand or capacity from destabilising the price. Would the hierarchy among the contracts change? The results of a numerical study are presented in the next section.

\subsection{Second numeric example: case of univariate stochastic process}

Let us consider now the case of a transport market where the spot price is fixed: $P=8$. In this case, the shipper's budget function is rewritten as

$$
B(n)=8 \times 1.2 \times \mu_{X} \times n .
$$

We deal with a univariate stochastic process consisting of just the demand faced by the shipper.

The calculations are relegated to Appendix F.

For a MPC to emerge as the favoured contract for both shipper and carrier, we need to set $2 \leq q \leq 12$, which leads to a fixed fee $0 \leq r \leq 23.09$ and $5.984 \leq s \leq 8.498$. For this to happen, we have as well $8.20<p_{r} \leq 9.6$ and the corresponding vectors $\{\alpha, \beta, \theta\}$ which enable Condition 6 to be satisfied.

For a QFC to emerge as a dominant contract, we must have $q=1,8.2 \leq p_{r} \leq 9.6$ and the other parameters of the QFC as required for its existence. 
We recapitulate the results in Table 3.

As can be seen, the MPC also has good chances to be retained. This is due to the fact that the lesser variance of the spot market enables the shipper to retain a larger proportion of her budget, whatever commitment she agrees with the carrier. If the carrier can force the shipper into accepting his terms, then he is better off overall in picking a MPC with $q=11$.

The choice of contract parameters presented in the table do not do justice to the range of available values which still yield the same profit or retained budget, but the calculations involved (in all, 5 parameters can change independently: $q, r, \beta, \alpha$ and $\theta$ ) were too complex and too large, so we chose to just present some values of interest. Note that given the alternatives of the QFC or PRC, to retain the MPC when the choice is endogenous, the shipper has to accept to push her commitment up to $q=11$ thus enabling the carrier to make a large profit of 1832, even with $r=0.01$.

These results still beg the question of when and why the shipper and carrier would choose one contractual form or another in a more general context. To address these issues, we take now a look at what would happen if both took recourse in the spot market and the impact of the variance of both the spot market price and demand. We enlarge the scope of the results by first evaluating the cost and opportunity for shipper and carrier to engage in a pure spot market sourcing strategy in the next section before addressing the issue of the variance of all four strategies in the section after that.

Table 3 Univariate case: table of maximum contract outcomes for each player separately and parameter conditions for exogenous or endogenous choice of contract

\begin{tabular}{|c|c|c|c|c|}
\hline \multirow[b]{2}{*}{$n=40$} & \multicolumn{2}{|c|}{ Carrier } & \multicolumn{2}{|c|}{ Shipper } \\
\hline & Exogenous & Endogenous & Exogenous & Endogenous \\
\hline \multirow[t]{2}{*}{ PRC } & 2320 & 2320 & 2320 & 2320 \\
\hline & $p_{r}=9.6$ & $p_{r}=9.6$ & $p_{r}=3.8, q=1$ & $p_{r}=3.8, q=1$ \\
\hline \multirow[t]{2}{*}{ MPC } & 2974 & 2849 & 923.6 & 923.6 \\
\hline & $\begin{array}{l}q=12, r=0 \\
s=8.49\end{array}$ & $\begin{array}{l}q=11, r=13.03 \\
s=7.11\end{array}$ & $\begin{array}{l}q=8, s=5.98 \\
r=0\end{array}$ & $\begin{array}{l}q=8, s=5.98 \\
r=0\end{array}$ \\
\hline \multirow[t]{2}{*}{ QFC } & 2320 & 644.9 & 2320 & 1675 \\
\hline & $\begin{aligned} \alpha & =9.3 \\
\beta & =400, \theta=0\end{aligned}$ & $\begin{array}{l}\alpha=15.68, \beta=400 \\
\theta=1.558\end{array}$ & $\begin{array}{l}\alpha=0 \\
\beta=400, \theta=0\end{array}$ & $\begin{array}{l}\alpha=15.898, \beta=400 \\
\theta=0, q=1\end{array}$ \\
\hline
\end{tabular}

\subsection{Third numerical example: pure spot procurement strategy}

Let us now consider the case of the shipper who decides to forego a contract with a carrier and decides to procure all his transport needs directly from the spot market.

In this example, we are very near to the supply chain management case presented in $\mathrm{Wu}$ et al. (2002) where a buyer has the possibility of combining procurement from a contract and from a spot market to resolve his necessities. In that paper's model, the buyer can choose to privilege either the contract or the spot market, depending on the relative cost of either. However, since demand is modelled as being a function of spot market price, we feel that the answers provided in that paper do not reflect the real world practice of transport, where, as seen before, spot market prices may be correlated with demand by a factor $\rho$ and $0<\rho<1$. 
The shipper buying exclusively from the spot market her transport capacity requirements can be considered as a particular case of a MPC where both the commitment $q$ and the fixed fee $r$ are set to nought. The profit function and expected profit of the shipper can be written as

$$
\begin{aligned}
V_{\text {Spot }}\left(x_{t}, p_{t}\right) & =B(n)-x_{t} p_{t} \\
E_{\text {Spot }}^{s} & =B-n\left[I+\mu_{X} \mu_{P}+\operatorname{Cov}(X, P)\right] \\
& =B(n)-n\left[I+\mu_{X} \mu_{P}+\sigma_{X} \sigma_{P} \rho\right] .
\end{aligned}
$$

When we evaluate it for $n=40$, we obtain an expected residual budget of 470 .

In the case of the carrier, the profit to be had by simply selling all his available capacity on the spot market yields 1680 since he does not incur the information cost which affects the shipper. Both results are included in Table 2.

As mentioned earlier, we do not consider these alternative strategies as viable in the long term because of considerations which are difficult to include in the present model, notably the liquidity of the spot market on a long term basis. Another issue is the variance of the cost or the profit to be had in this type of transaction. We now turn our attention to it by evaluating the variance of residual budget or profit for all strategies previously presented.

\subsection{Variance of results from contracts for shipper and carrier}

In effect, if the shipper decides to source all her transport requirements from the spot market, she is letting herself be affected by the variance of the spot market price. If as is often the case, she must respect an overall budget for this cost, this variance is bound to substantially impact it.

The calculations of the variance of the three contracts and the variance of the spot market are relegated to Appendix G.

We find that the variance of spot market procurement is equal to 1186.3 over just one period.

Table 4 Table of maxima and minima of the variances of the different transport procurement strategies according to the capacity commitment over 40 periods (bivariate demand and spot market)

\begin{tabular}{lll}
\hline \multirow{2}{*}{$\begin{array}{l}\text { Procurement } \\
\text { strategy }\end{array}$} & \multicolumn{3}{c}{ Variance $\left(\times 10^{6}\right)$} \\
\cline { 2 - 3 } Spot & Max & Min \\
PRC & 1.898 & 1.898 \\
& 1.327 & 0.130 \\
MPC & $p_{r}=9.6$ & $p_{r}=3.8$ \\
& 1.774 & 0.723 \\
QFC & $q=1$ & $q=10$ \\
& 0.840 & 0.145 \\
& $\beta=320$ & $\beta=448$ \\
& $\alpha=0$ & $\alpha=1$ \\
& $\theta=0$ & $\theta=0.1$ \\
\hline
\end{tabular}


We compare the three contracts over 40 periods. The results are tabulated in Table 4 . As can be observed, the possibility of contracting ex ante transport capacity clearly exhibits important differences in terms of the variance of the cost of transport for the shipper. The most favourable contract being the QFC, a result which is intuitive: the shipper reduces uncertainty in both the cost of transport and the uncertainty of demand by contracting in advance the required transport capacity over a number of periods. The variance is even lower than the MPC's on all capacity commitments. This is due to the length of the period considered here $(n=40)$ : for lesser lengths of time, the result would not be so clear cut. This difference increases when increasing $n$, favouring the QFC contract when the shipper has the ability to contract over multiple periods.

\section{References}

Baker, G., Gibbons, R. and Murphy, K. (2001) 'Bringing the market inside the firm?', American Economic Review, Vol. 91, No. 2, pp.212-218.

Baker, G., Gibbons, R. and Murphy, K. (2002) 'Relational contracts and the theory of the firm', Quarterly Journal of Economics, Vol. 117, No. 1, pp.39-83.

Brusset, X. and Temme, N.M. (2005) 'Supply chain management - european perspectives', Impact of Information and Coordination on Transport Procurement, 1st ed., Chap. 12, Copenhagen Business School Press, Copenhagen, Denmark, pp.239-261.

Cachon, G. and Lariviere, M. (2001) 'Contracting to assure supply: how to share demand forecasts in a supply chain', Management Science, Vol. 47, No. 5, pp.629-646.

Chen, F. (2004) 'Information sharing and supply chain coordination', in de Kok, T. and Graves, S. (Eds.): Handbooks in Operations Research and Management Science: Supply Chain Management, Vol. 11, Chap. 7, Elsevier, pp.341-421.

Corbett, C., Zhou, D. and Tang, C. (2004) 'Designing supply contracts: a contract type and information asymmetry', Management Science, Vol. 50, No. 4, pp.550-559.

Jackson, M.O. and Wilkie, S. (2005) 'Endogenous games and mechanisms: side payments among players', Review of Economic Studies, Vol. 72, pp.543-566.

Kleindorfer, P.R. and Wu, D.J. (2003) 'Integrating long- and short-term contracting via business-to-business exchanges for capital-intensive industries', Management Science, Vol. 49, No. 11, pp.1597-1615.

Knemeyer, A.M., Corsi, T. and Murphy, P.R. (2003) 'Logistics outsourcing relationships: customer perspectives', Journal of Business Logistics, Vol. 24, No. 1, pp.77-109.

Laffont, J.J. and Tirole, J. (1993) A Theory of Incentives in Procurement and Regulation, MIT Press, Cambridge, MA, pp.82-83.

Lagunoff, R. (1992) 'Fully endogenous mechanism selection: an essay on endogenous institutional choice', Economic Theory, Vol. 2, No. 4, pp.465-480, available at http://ideas.repec.org/a/spr/joecth/v2y1992i4p465-80.html

Lariviere, M. (1999) 'Supply chain contracting and coordination with stochastic demand', in Tayur, S., Ganeshan, R. and Magazine, M.(Eds.): Quantitative Models for Supply Chain Management, Chap. 8, Kluwer Academic, Dordrecht, Holland, pp.234-267.

Lovejoy, W.S. (2006) 'Optimal mechanisms with finite agent types', Management Science, Vol. 52, No. 5, pp.788-803.

Macauley, S. (1963) 'Non-conctractual relations in business', American Sociological Review, Vol. 28, No. 1, pp.55-67. 
Martínez-de-Albéniz, V. and Simchi-Levi, D. (2005) 'A portfolio approach to procurement contracts', Production and Operations Management, Vol. 14, No. 1, pp.90-114.

Myerson, R.B. (1979) 'Incentive compatibility and the bargaining problem', Econometrica, Vol. 47, No. 1, pp.61-73.

Özalp, O. and Wei, W. (2006) 'Strategic commitment for optimal capacity decision under asymmetric forecast information', Management Science, Vol. 52, No. 8, pp.1238-1257.

Plambeck, E.L. and Taylor, T.A. (2005) 'Sell the plant? The impact of contract manufacturing on innovation, capacity, and profitability', Management Science, Vol. 51, No. 1, pp.133-150.

Plambeck, E.L. and Taylor, T.A. (2007) 'Supply chain relationships and contracts: the impact of repeated interaction on capacity investment and procurement', Management Science, Vol. 53, No. 10, pp.1577-1593.

Reichheld, F.F. and Sasser Jr., W.E. (1993) 'Loyalty-based management', Harvard Business Review, Vol. 71, No. 2, pp.64-73.

Rubinstein, A. (1982) 'Perfect equilibrium in a bargaining model', Econometrica, Vol. 54, pp.97-109.

Salanié, B. (1997) The Economics of Contracts: A Primer, 1st ed., MIT Press, Cambridge, MA, pp.97-116.

Seifert, R., Thonemann, U. and Hausman, W. (2004) 'Optimal procurement strategies for online spot markets', European Journal of Operational Research, Vol. 152, No. 3, pp.781-799.

Spinler, S. and Huchzermeier, A. (2006) 'The valuation of options on capacity with cost and demand uncertainty', European Journal of Operational Research, Vol. 171, No. 3, pp.915-934.

Tomlin, B. (2003) 'Capacity investments in supply chains: sharing the gain rather than sharing the pain', Manufacturing and Service Operations Management, Vol. 5, No. 4, pp.317-333.

Tsay, A. (1999) 'Quantity flexibility contract and supplier-customer incentives', Management Science, Vol. 45, No. 10, pp.1339-1358.

Tsay, A. and Lovejoy, W. (1999) 'Quantity flexibility contracts and supply chain performance', Manufacturing and Service Operations Management, Vol. 1, No. 2, pp.89-111.

Tsay, A., Nahmias, S. and Agrawal, N. (1999) 'Quantitative models for supply chain management', International Series in Operations Research and Management Science, 1st ed., Chap. 10, Modeling Supply Chain Contracts: A Review, Kluwer Academic, pp.299-336.

Wu, D.J. and Kleindorfer, P. (2003) 'Competitive options, supply contracting and electronic markets’, Management Science, Vol. 51, No. 3, pp.452-466.

$\mathrm{Wu}$, D.J., Kleindorfer, P.R. and Zhang, J.E. (2001) 'Optimal bidding and contracting strategies in the deregulated electric power marketplace: Part II', in Sprague Jr., R.H. (Ed.): Proceedings of the 34th Annual Hawaii International Conference on Systems Sciences, IEEE Computer Society Press, Los Alamitos, California, USA, pp.2033-2043.

Wu, D.J., Kleindorfer, P.R. and Zhang, J.E. (2002) 'Optimal bidding and contracting strategies for capital-intensive goods', European Journal of Operational Research, Vol. 137, No. 3, pp.657-676.

Wu, D.J., Kleindorfer, P.R. and Sun, Y. (2005) 'Optimal capacity expansion in the presence of capacity options', Decision Support Systems, Vol. 40, Nos. 3-4, pp.553-561. 


\section{Notes}

${ }^{1}$ In the process, each game results from the choice of a game which in turn results from the choice from another game, etc.

${ }^{2}$ The supplier is in fact trying to coax the buyer into using this available capacity.

${ }^{3}$ This is the case for example for non-dangerous palletised products and tautliners: loads or trucks can be exchanged among themselves.

${ }^{4}$ This situation can be contrasted with Spinler and Huchzermeier (2006), Kleindorfer and Wu (2003) where demand is an inverse function of the spot price, but is similar to the setting in Seifert et al. (2004).

${ }^{5}$ In contrast to the assumptions underlying the model in Spinler and Huchzermeier (2006).

${ }^{6}$ This effect is similar to the 'learning curve' effect: as the number of games runs up, the information effect represents a rent to the carrier which he can hide from the shipper.

${ }^{7}$ This type of objective function is introduced in the 1950 s by Markowitz within the Mean-Variance Theory, basis of the modern portfolio selection theory.

${ }^{8}$ This contract is on purpose very similar to the option contract presented in Spinler and Huchzermeier (2006).

${ }^{9}$ See the definition for $\mathrm{S}(\mathrm{K})$ presented in Section 2 of Cachon and Lariviere (2001).

${ }^{10}$ See the discussion in Appendix E.

${ }^{11}$ The reasons for this penalty is to ensure that the shipper conveys demand forecasts in a credible way. Note that in this contract no other lump payment is made which would otherwise ensure such credibility. The reader can refer to Chen (2004) and Cachon and Lariviere (2001) among others for further justifications.

${ }^{12} \mathrm{We}$ are interested in feasible and tractable solutions, not mathematical proofs.

${ }^{13}$ Unless this is the $N$ th game, in which case the sunk investments have come to the end of their useful lives.

${ }^{14}$ The details of all calculations are available upon request to the author.

${ }^{15}$ I.e., where both differentials in terms of $n$ are positive.

\section{Appendix}

\section{A Proof of Theorem}

Proof of Theorem 2: There is an optimal commitment for the shipper or the carrier if the shipper's or the carrier's objective function are concave. For the shipper, this is the case iff

$$
\frac{\partial^{2} E_{s}^{2}(q, s, r, n)}{\partial q^{2}}=s f_{X}(q)+I f_{X}^{\prime}(q)-\frac{\partial^{2} \varphi_{s}(q)}{\partial q^{2}} \leq 0 .
$$

We look for the optimal quantity $q$ which should satisfy both players.

$$
\frac{\partial E_{s}^{2}(q, s, r)}{\partial q}=-s \overline{F_{X}}(q)+I f_{X}(q)-\frac{\partial \varphi^{s}(q)}{\partial q} .
$$

The F.O.C. requires that

$$
s=\frac{I f_{X}(q)-\varphi^{s \prime}(q)}{\overline{F_{X}}(q)},
$$

subject to $F_{X}(q)<1$ and the shipper's objective function being concave. 
For the carrier, the first differential in $q$ of his profit function is written

$$
\begin{aligned}
& \frac{\partial E_{c}^{2}(q, s, r)}{\partial q}=(s-c) \overline{F_{X}}(q)+\frac{\partial \varphi^{c}(q)}{\partial q} . \\
& \frac{\partial^{2} E_{c}^{2}(q, s, r)}{\partial q^{2}}=-(s-c) f_{X}(q)+\frac{\partial^{2} \varphi^{c}(q)}{\partial q^{2}} .
\end{aligned}
$$

The second derivative of $\varphi(q)$ may not be always negative for all $q$ so that no conclusion can be drawn as to whether the extremum in $q$ for the carrier is actually an optimum. In point of fact, in the bivariate example presented here, the values of $q$ which mark local extremums are minimums.

Depending on the bargaining power of both players, the final commitment on which both agree may be equal to the optimum of the shipper or higher.

\section{B Objective functions with a QFC}

Given that all periods of the game are symmetric: each demand outcome is i.i.d. with respect to the others and the spot prices are also i.i.d. with respect to the other spot prices. We can reorder the realised demands within the overall sum in each of the objective functions of the buyer and supplier irrespective of what demand occurs exactly in which period.

The objective functions take different forms whether the sum of outcome demands is higher than $\beta$ or not: if the shipper has overestimated her demand, she has to pay a penalty.

$$
\begin{aligned}
& \beta \geq \sum_{i=1}^{i} x_{i}, \quad\left\{\begin{array}{l}
V_{3}(X, P)=\alpha \sum_{i=1}^{n} x_{i}+\theta\left(\beta-\sum_{i=1}^{n} x_{i}\right) \\
\pi_{3}(X, P)=\alpha \sum_{i=1}^{n} x_{i}+\theta\left(\beta-\sum_{i=1}^{n} x_{i}\right)-c \sum_{t=1}^{n} x_{i}-n K
\end{array}\right. \\
& \beta<\sum_{i=1}^{n} x_{i}, \quad \exists j \mid j \leq n \wedge \sum_{i=1}^{n-j-1} x_{i} \leq \beta \wedge \sum_{i=1}^{n-j} x_{i}>\beta, \\
& \pi_{3}(X, P)=\alpha \beta+\left(\sum_{i=1}^{n-j} x_{i}-\beta\right) p_{n-j}+\mu_{P} \sum_{i=n-j+1}^{t} x_{i}-c \sum_{i=1}^{n} x_{i}-n K .
\end{aligned}
$$

To evaluate the dispersion of demands around $\beta$, we need to calculate the variance of the sum of demands within a game. Because demand is a stationary stochastic process, its sum over $n$ periods is also a stationary process, and its variance is finite. Let us call $Y_{n}=\sum_{i=1}^{n} x_{i}$. By the central limit theorem, for a large $n$,

$$
Y_{n} \sim \mathcal{N}\left(n \mu_{X}, \sqrt{n} \sigma_{X}\right)
$$

Let us call $f_{Y}($.$) and F_{Y}($.$) the pdf and cdf of this normal distribution. Let us restrain$ the present study to the support [0,QY $Y_{H i}$, with $Q Y_{H i}$ a suitably high number. 
In the following, we shall assume that $n$ is sufficiently large as to render the Central Limit Theorem applicable.

\section{Expected optimal parameters in the QFC}

The parameters of the contract $\alpha, \beta, \theta$ and $n$ presented in Condition 4 are discussed and evaluated.

The shipper is interested in minimising the impact of both the spot market when she underestimates the demand she has to satisfy and the penalty she has to pay when she overestimates the demand.

\section{A Optimal $\theta$ :}

$$
\frac{\partial E_{s}^{3}(\alpha, \beta, \theta, n)}{\partial \theta}=-\left(\frac{\beta}{F_{Y_{n}}(\beta)}-g_{1}(\beta, n)\right) \leq 0 .
$$

So the shipper is always looking to minimise the penalty. By the same token, the carrier is always looking to increase it.

The shipper would not accept a contract in which the penalty for not forecasting correctly the demand to be carried over the length of the contract is equal to the whole budget she has identified for transport. The carrier would not countenance a penalty where he would have to actually pay for a forecasting error imputable to the shipper.

So, the range for this parameter is

$$
0 \leq \theta \leq \frac{B-n \alpha \mu_{X}-\mu_{P}\left(g_{2}(\beta, n)-\beta / \overline{F_{Y_{n}}}(\beta)\right)}{\beta / F_{Y_{n}}(\beta)-g_{1}(\beta, n)} .
$$

The higher the penalty, the higher the confidence that the shipper has in her forecasts (meaning she has a pretty good notion that she won't have to pay up), or the higher the carrier's bargaining power, or both. This commitment by the shipper resembles the commitment presented in the model in Cachon and Lariviere (2001). A large penalty clearly signals that the shipper expects to exceed her transport commitment.

\section{B Optimal $\beta$ :}

The differential in terms of $\beta$ of the shipper's and carrier's objective functions are only different in sign:

$$
\begin{aligned}
\frac{\partial \Psi(\beta, \theta, n)}{\partial \beta}= & \theta\left(\frac{1}{F_{Y_{n}}(\beta)}-\frac{\beta f_{Y_{n}}(\beta)}{F_{Y_{n}}(\beta)^{2}}-\frac{\partial g_{1}(\beta, n)}{\partial \beta}\right) \\
& +\mu_{P}\left(\frac{\partial g_{2}(\beta, n)}{\partial \beta}-\frac{1}{\overline{F_{Y_{n}}}(\beta)}+\frac{\beta f_{Y_{n}}(\beta)}{\overline{F_{Y_{n}}}(\beta)^{2}}\right) .
\end{aligned}
$$

Whatever the type of distribution of demands, $g_{1}, g_{2}$ are always continuous on the domain of interest, $\theta \geq 0$ so $\Psi(\beta, \theta, n)$ is also continuous and may admit an extremum over the domain. However, by construction, this will be a maximum for one party when it is a minimum for the other party.

The shipper will be looking for a low $\beta$ when the carrier looks for a high one: we have no way of telling how the bargaining will turn. 


\section{Optimal n:}

As an initial remark, the reader is reminded that both carrier and shipper are involved in a transaction setting which requires an initial investment which has yet to run its full economic life. This life is a function of the number of games that both participants play but not of the length of each game. Both are motivated to increase the lifetime of the investments because at the end of the useful life of those investments, they will have to incur new ones. So both are inclined to choose $n$ as large as possible.

To evaluate the optimal number of periods, we linearise $n$ and differentiate according to it. We obtain

$$
\frac{\partial E_{s}^{3}(\alpha, \beta, \theta, \tilde{n})}{\partial \tilde{n}}=\frac{\partial B(\tilde{n})}{\partial \tilde{n}}-\alpha \mu_{X}-\frac{\partial \Psi(\beta, \theta, \tilde{n})}{\partial \tilde{n}} .
$$

The optimal $n$ to the shipper is when the F.O.C. is satisfied, meaning that

$$
\alpha=\frac{1}{\mu_{X}}\left[\frac{\partial B(\tilde{n})}{\partial \tilde{n}}-\frac{\partial \Psi(\beta, \theta, \tilde{n})}{\partial \tilde{n}}\right] .
$$

The equivalent optimal parameter for the carrier is different unless $\partial B(n) / \partial n=$ $K+c \mu_{X}$, which has no reason to be. So the parameter $\alpha$, result of the bargaining, will fall within a range defined by the regions in which both partners wish to extend the number of periods. ${ }^{15}$

So, both players are likely to choose $\alpha$ such that

$$
\begin{aligned}
& \alpha \leq \frac{1}{\mu_{X}}\left[\frac{\partial B(\tilde{n})}{\partial \tilde{n}}-\frac{\partial \Psi(\beta, \theta, \tilde{n})}{\partial \tilde{n}}\right] \\
& \alpha \geq c+\frac{K}{\mu_{X}}-\frac{1}{\mu_{X}} \frac{\partial \Psi(\beta, \theta, \tilde{n})}{\partial \tilde{n}} .
\end{aligned}
$$

Along the way we obtain a 'desirable' range for $\alpha$, something which is not given from the F.O.C. in $\alpha$ of the players' objective functions since the carrier's first differential in $\alpha$ of his objective function is always positive and the shipper's is always negative. However, this range is not mandatory: choosing a value outside this range does not impede the players from reaching an agreement, so we shall only mention it here but not include it in the set of conditions for the eligibility of the contract.

\section{Participation constraints}

The participation constraints on the players also require that

$$
\left\{\begin{array}{l}
E_{3}^{s}(\alpha, \beta, \theta, \tilde{n}) \geq 0 \\
E_{3}^{c}(\alpha, \beta, \theta, \tilde{n}) \geq 0 .
\end{array}\right.
$$

with

$$
\begin{aligned}
& E_{3}^{s}(\alpha, \beta, \theta, \tilde{n})=B(\tilde{n})-\tilde{n} \alpha \mu_{X}-\Psi(\beta, \theta, \tilde{n}) \\
& E_{3}^{c}(\alpha, \beta, \theta, \tilde{n})=\tilde{n}\left[\alpha \mu_{X}-c \mu_{X}-K\right]+\Psi(\beta, \theta, \tilde{n}) .
\end{aligned}
$$


By replacing function $\Psi$ by its definition in (17), the participation constraints can now be written

$$
\begin{aligned}
& \theta\left(\frac{\beta}{F_{Y_{n}}(\beta)}-g_{1}(\beta, \tilde{n})\right) \leq B(\tilde{n})-\tilde{n} \alpha \mu_{X}-\mu_{P}\left(g_{2}(\beta, \tilde{n})-\frac{\beta}{\overline{F_{Y_{n}}}(\beta)}\right) \\
& \theta\left(\frac{\beta}{F_{Y_{n}}(\beta)}-g_{1}(\beta, \tilde{n})\right) \geq \tilde{n} K+\tilde{n} c \mu_{X}-\tilde{n} \alpha \mu_{X}-\mu_{P}\left(g_{2}(\beta, \tilde{n})-\frac{\beta}{\overline{F_{Y_{n}}}(\beta)}\right)
\end{aligned}
$$

The Figure 2 represents the area where the tuples $\{\alpha, \beta\}$ with both $\alpha$ and $\beta$ positive enable a positive penalty $\theta$ to exist in the numerical example of a bivariate demand and price.

Because all the above functions and expressions are continuous and do not change when we consider integer values for $n$, in the following we shall drop the tilde over the $n$ and consider that we are using whole periods only.

We can now enunciate the conditions of existence of the QFC:

$$
\left\{\begin{array}{l}
\alpha \geq 0, \wedge \theta \geq 0, \wedge \beta>0 \\
\theta \leq \frac{1}{\beta / F_{Y_{n}}(\beta)-g_{1}(\beta, n)}\left[B(n)-n \alpha \mu_{X}-\mu_{P}\left(g_{2}(\beta, n)-\beta\right)\right] \\
\theta \geq \frac{1}{\beta / F_{Y_{n}}(\beta)-g_{1}(\beta, n)}\left[n K+n c \mu_{X}-n \alpha \mu_{X}-\mu_{P}\left(g_{2}(\beta, n)-\beta\right)\right] .
\end{array}\right.
$$

Figure 2 The grey segments represent the possible tuples $\{\beta, \alpha\}$ for which a QFC which coordinates the shipper and carrier can be chosen in the bivariate numerical example developed later (see online version for colours)

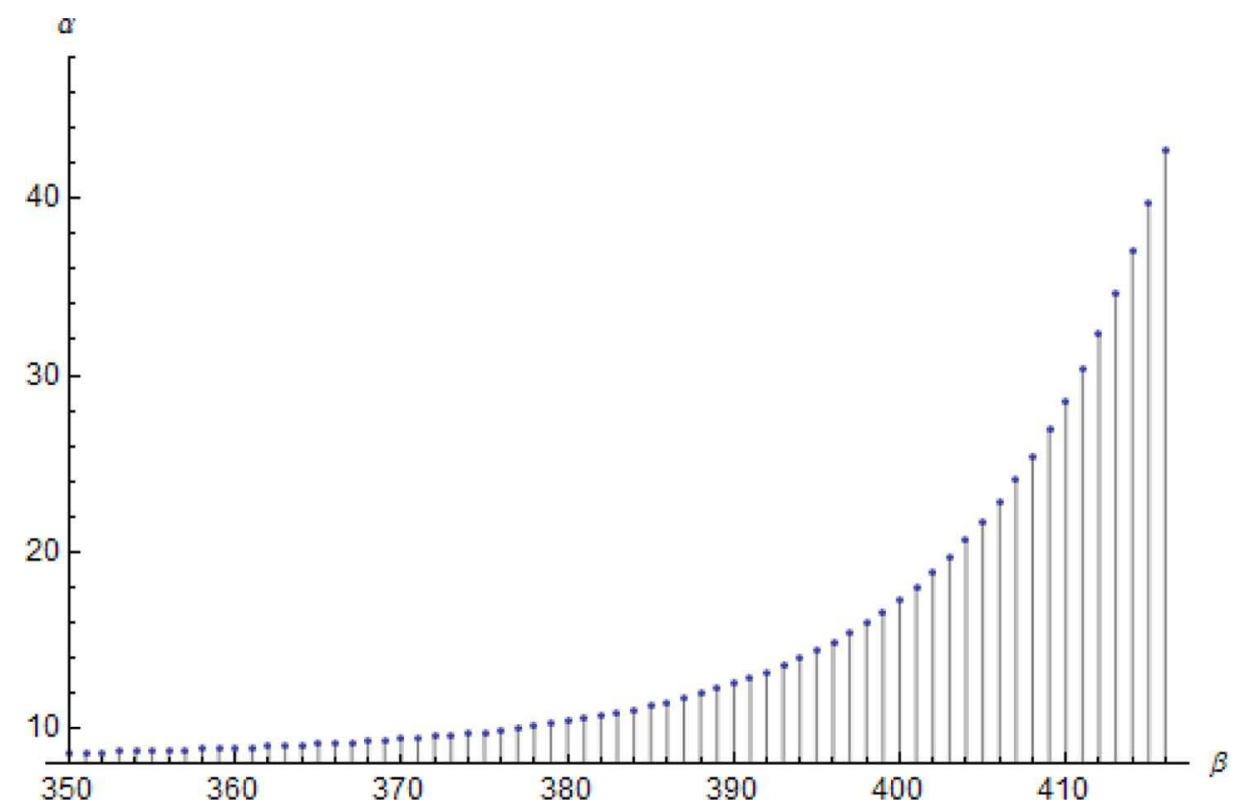




\section{Proof of Condition 7}

Proof: From (28) and for both players to choose a QFC it must generate more reward than the MPC to each player. Since $n>0$, we must have

$$
\left\{\begin{array}{l}
r+s \mu_{X}(q)+I \overline{F_{X}}(q)+\varphi^{s}(q) \geq \Psi(\beta, \theta, n) / n+\mu_{X} \alpha \\
c \mu_{X}+r+(s-c) \mu_{X}(q)+\varphi^{c}(q) \leq \Psi(\beta, \theta, n) / n+\mu_{X} \alpha .
\end{array}\right.
$$

Further, the parameters $r, s$ and $q$ have to satisfy Condition 3 and $\beta, \theta$ and $n$ must satisfy Condition 4.

\section{E Numeric example with bivariate variables}

\section{A Conditions for a PRC to exist}

We first start by determining the conditions for a PRC from Condition 1 to be eligible, $p_{r}$ has to satisfy

$$
3.80 \leq p_{r} \leq 9.6 .
$$

\section{$B$ Condition for a MPC to exist (Condition 3)}

To know the MPC to be satisfactory per se for both players, let us look at how to satisfy Theorem 2, that is to say, which values of $q$ enable an optimal $s$ to satisfy both players simultaneously.

We notice that the shipper's objective function is not concave: the second differential in $q$ of the objective function of the shipper is not always negative, so we cannot apply here the Theorem 2 for the shipper. The same is true of the carrier's objective function, which precludes using a simple unique function to calculate the parameter $s$. We plot in Figure 3 the curves of values of $s$ in terms of $q$ : (a) the maximum values of $s$ over which the shipper will have eaten her budget, even if $r=0$, (b) the minimum value of $s$ for the carrier under which he cannot make a profit when $r=0$, (c) the curve under which the second differential of the shipper's objective function is negative, (d) the curve of $s(q)$.

For the sake of clarity, we assume here that the shipper and carrier will agree on a value of $s$ which satisfies Theorem 2 as long as $s$ does not violate the shipper's participation constraint and then follows the participation constraint limit.

The plot of the corresponding values of $s$ are given in Figure 4.

From the first two inequalities in Condition 3, we obtain as the grey segments in Figure 5 the tuples $\{q, r\}$ with a strictly positive fixed fee, meaning a fee that the shipper pays to the carrier. This limits $q$ to $1 \leq q \leq 11$.

Why does the fixed fee have to be strictly positive? We observe that the results presented in Cachon and Lariviere (2001) condition the coordination of the shipper and the carrier to the fact that the shipper signals her demand forecast credibly by paying ex ante the carrier a fixed fee. Only promising a higher per unit fee $s$ does not signal credibly the required capacity by the shipper, as shown in Cachon and Lariviere (2001). 
Figure 3 The thick lines are the upper and lower limits on $s$ for shipper and carrier to participate, the thin dotted line is the values of $s$ satisfying Theorem 2,

the thick dotted line limits the area where $\frac{\partial^{2} E_{2}^{s}}{\partial q^{2}}=0$ : under the line the differential is negative

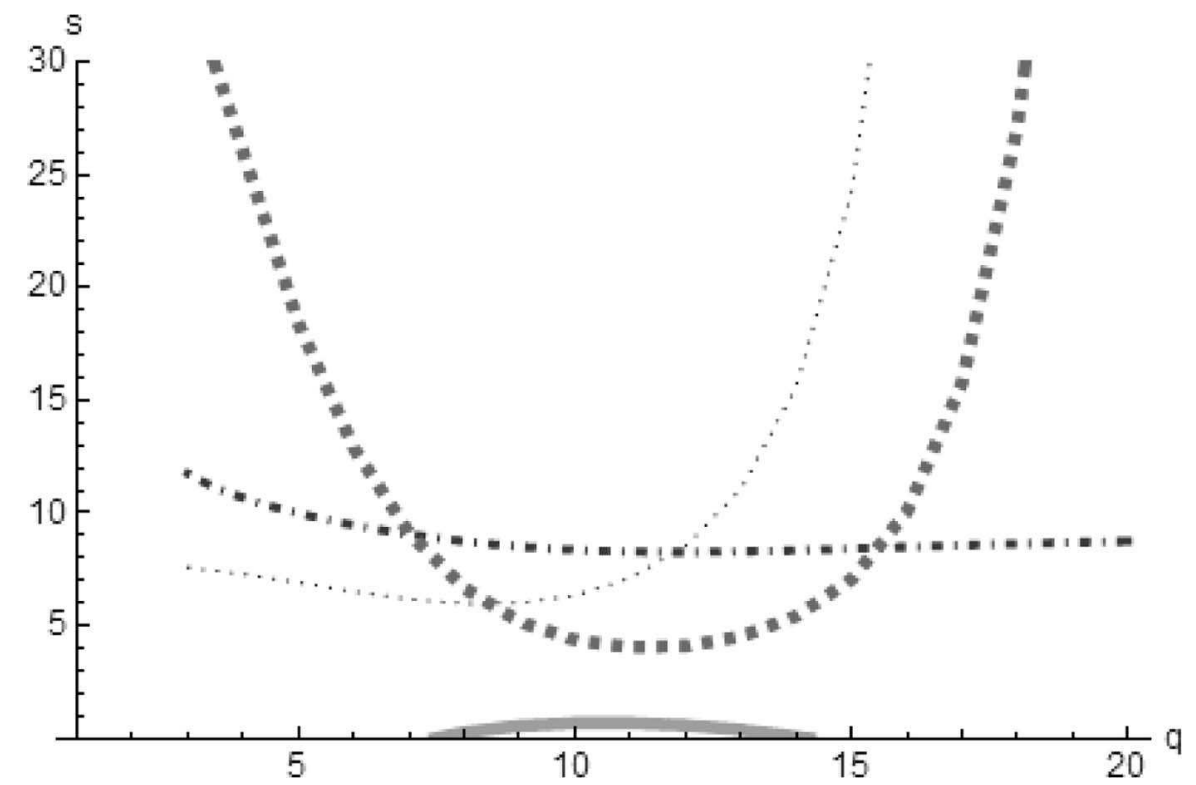

Figure 4 The values of $s$ in $q$ which satisfy Theorem 2 and respect the participation constraints, the thick dotted line limits the area where $\frac{\partial^{2} E_{2}^{s}}{\partial q^{2}}=0$ : under the line the differential is negative (see online version for colours)

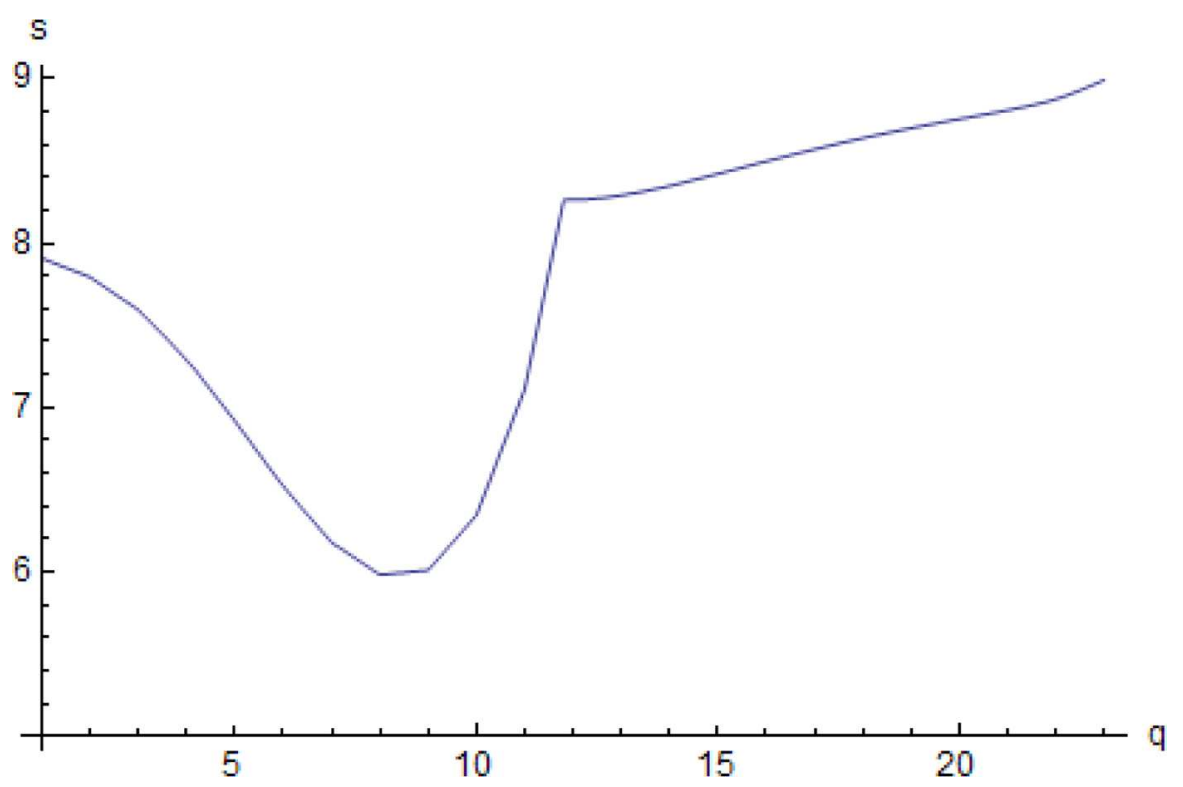


Figure 5 The segments represent the possible tuples $\{q, r\}$ for which a MPC which coordinates the shipper and carrier can be chosen. Parameter $r$ ranges between 0 (excluded) and 20.84 (see online version for colours)

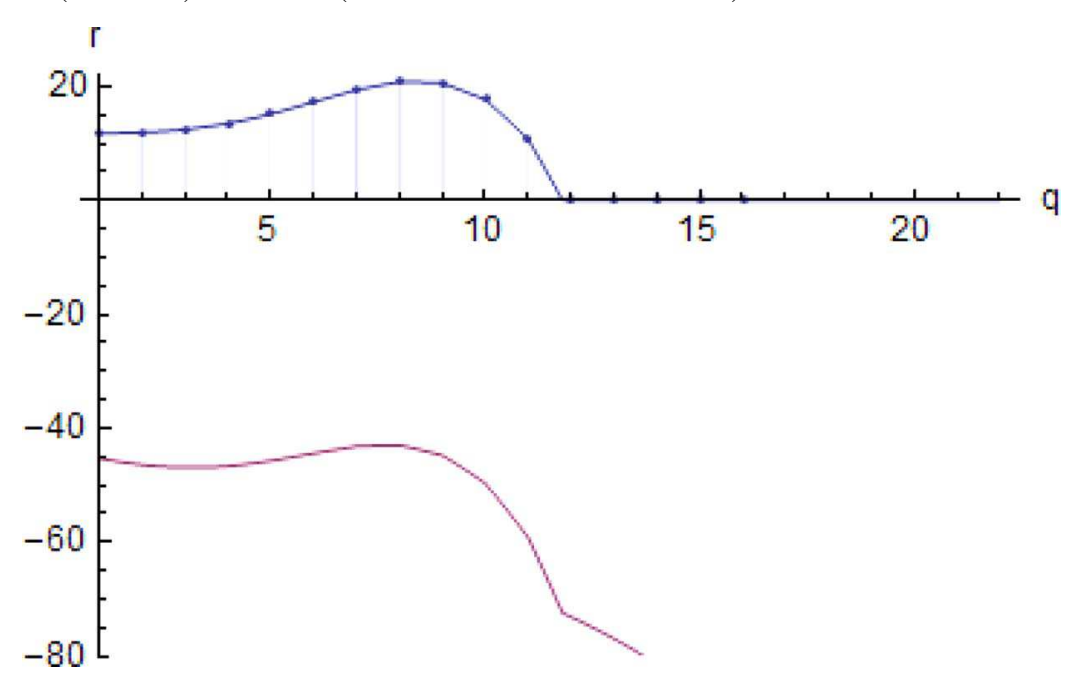

\section{Conditions for a QFC to exist (Condition 4)}

We first start by determining the conditions for a QFC alone. We then shall submit those to the comparison with conditions of the QFC dominating the other contracts.

How do we evaluate the number of periods which will satisfy both players? As we have seen in the considerations over the optimal $n$ in Appendix C, the conditions for both to agree on one value depend on their relative bargaining powers. So that the Central Limit Theorem can apply and without loss of generality, we assume that they agree on $n=40$.

The important constraints are the ones which determine $\theta$ as solution to the participation constraints expressed in terms of $\beta, \alpha$ and $n$ in Condition 4.

In Figure 6 the possible values for parameter $\theta$ are the positive values between the two envelopes which represent the upper and lower participation constraints, expressed in terms of $\alpha$ and $\beta$. As can be seen, there are upper limits on $\alpha$ which, whatever the value of $\beta$ within the domain, do not enable a contract to fulfill the participation constraints because the corresponding penalty would have to be set at a negative value. We shall consider only the vectors of parameters $\{\alpha, \beta, \theta\}$ for which the participation constraints are satisfied.

The corresponding solutions are an equilibrium in which the QFC is chosen in isolation, what would the outcome be if the shipper and carrier could compare it to the PRC?

\section{$D$ Condition for the QFC to be equivalent to the PRC (Condition 6)}

We join the constraints from Conditions 4 and from 6 . We evaluate $p_{r}$ when function $\Psi(\alpha, \beta, n)$ is replaced by its upper and lower bounds. 
Figure 6 The valid values of the parameter $\theta$ are the positive ones between the two envelopes in $\beta$ and $\alpha$ for Condition 4 to be fulfilled when $n=40$ (see online version for colours)

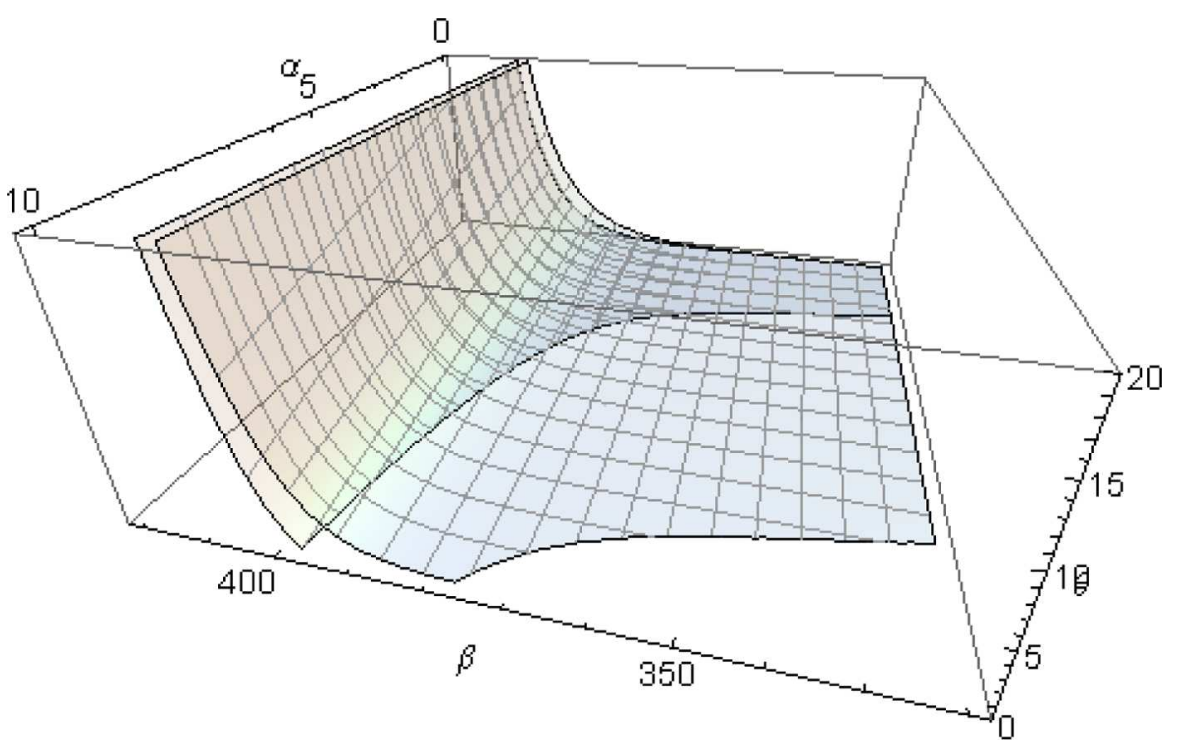

In Figure 7 we represent the upper and lower envelopes within which evolve the value of $p_{r}$ given tuples of $\{\beta, \alpha\}$ when $\theta$ is at or slightly higher than its low bound. When this is the case, the vectors $\alpha, \beta, p_{r}$ describe the volume between both grey envelopes. When $\theta$ takes on its high bound no values of $\beta$ and $\alpha$ can make a QFC compatible with a PRC.

However, we also have to satisfy the conditions of existence of the QFC in the first place. This is given by Condition 4 and as seen earlier (see Figure 6), not all tuples $\{\beta, \alpha\}$ comply. The limit on the combination of $\beta$ and $\alpha$ values are the same as the one for existence of a QFC in the first place.

Let us now look at the conditions of existence and dominance of a MPC over a PRC before submitting them to the comparison with the QFC.

\section{E Condition for a MPC to dominate a PRC (Condition 5)}

Let us consider how Condition 5 on the dominance of the MPC over the PRC can be met. We represent this set of inequalities as two functions in $q$ and $p_{r}$, as the high and low boundaries for $r$ in (22):

$$
\begin{aligned}
& \varrho_{H i}\left(q, p_{r}\right)=10 p_{r}-2 \overline{F_{X}}(q)-\varphi^{s}(q)-s(q) \mu_{X}(q) \\
& \varrho_{L o}\left(q, p_{r}\right)=10 p_{r}-\varphi^{c}(q)+1.8\left(\mu_{X}(q)-10\right)-s(q) \mu_{X}(q)
\end{aligned}
$$

with $s(q)$ as the function which generates $s$ from the values of $q$ as defined in $\mathrm{B}$. We observe that for $2 \leq q$, whatever value is given to $p_{r}$, we have $\varrho_{H i}\left(q, p_{r}\right)>$ $\varrho_{L o}\left(q, p_{r}\right)$, which satisfies the condition of preeminence of the MPC as set in (22) of Condition 5, so the MPC prevails for all $2 \leq q<12$. When $q=1$, we get $\varrho_{H i}\left(q, p_{r}\right)<$ $\varrho_{L o}\left(q, p_{r}\right)$, which means that the PRC is preferred to the MPC insofar as $r \geq 0$, as the 
inequality set (23) in Condition 5 is satisfied. by inspection, this means that we must have at least $p_{r} \geq 8.425$, otherwise we would have $r<\varrho_{L o}\left(1, p_{r}\right) \leq 0$ : the MPC under comparison would not be a coordinating one. Even though the players will not retain it, the outcome must be based on contracts which respect the constraints set up.

Figure 7 Between the envelopes which limit the value of $p_{r}$ are given vectors $\{\beta, \alpha, \theta\}$ for a QFC to be comparable to the PRC: the highest envelope is the one when $\theta$ assumes its high bound, the lower one is for the low bound on $\theta$, which at times may be equal to 0 (see online version for colours)

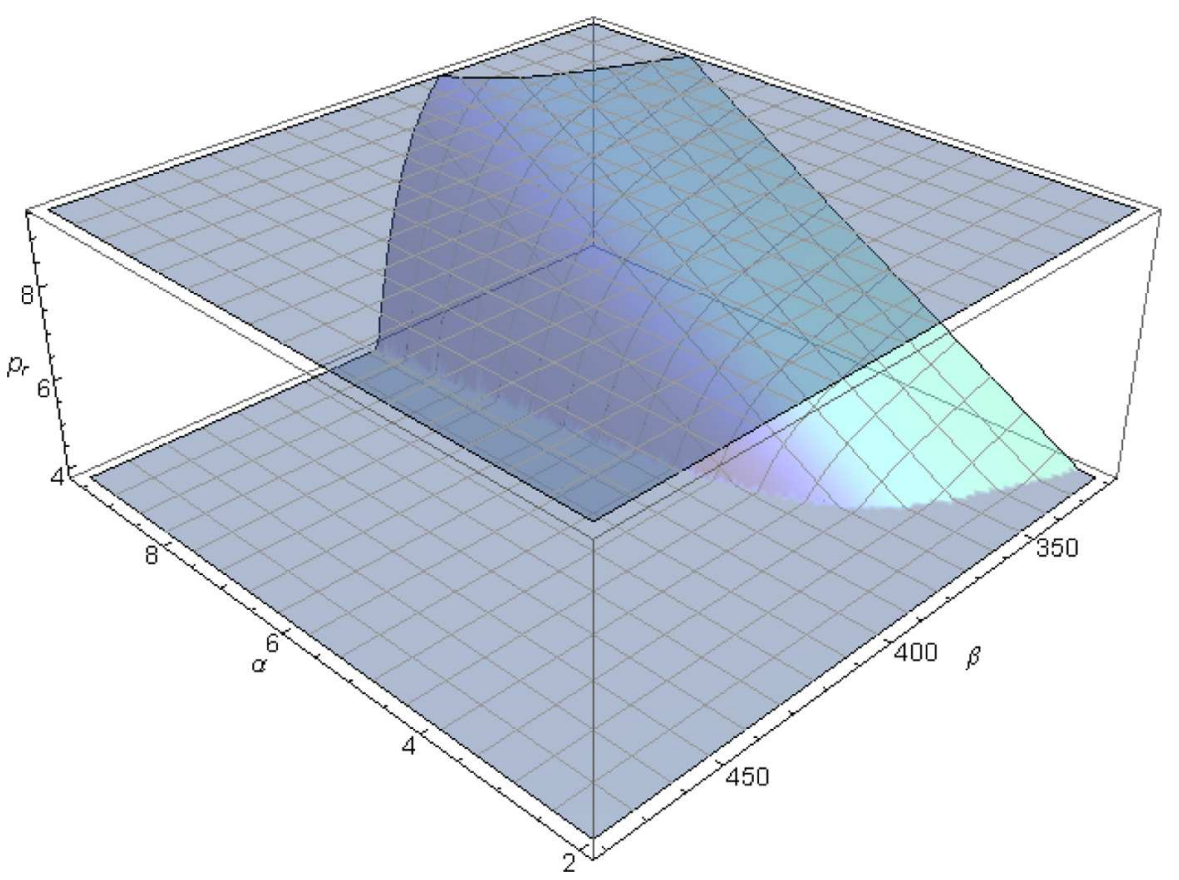

When $q \geq 12$, we observe that no possible positive value of $r$ can satisfy Condition 5: for those values of $q$, the MPC is no longer coordinating shipper and carrier so shall be discarded in this setting as of limited interest. Figure 8 presents the case of lower and higher limits for $r$ in terms of $p_{r}$ : not all values of $p_{r}$ can enable both players to be coordinated by a lump sum payment that the shipper makes to the carrier. When $p_{r} \leq 7.51$, the shipper would actually receive a payment from the carrier!

\section{F Condition for a QFC to dominate a MPC (Condition 7)}

We now look at the requirements so that the QFC dominate the MPC given in the set of inequalities (30) of Condition 7. Given that previous conditions of existence of the QFC also have to be satisfied, we obtain

$$
\left\{\begin{array}{l}
r+s \mu_{X}(q)+I \overline{F_{X}}(q)+\varphi^{s}(q)>\Psi_{H i}(\alpha, \beta, n) / n+\mu_{X} \alpha \\
c \mu_{X}+r+(s-c) \mu_{X}(q)+\varphi^{c}(q)<\Psi_{L o}(\alpha, \beta, n) / n+\mu_{X} \alpha
\end{array}\right.
$$


Figure 8 The shaded band above the abscissa represents the possible $r$ for the corresponding values of $p_{r}$ when $q=8$. The band widens progressively as $q$ increases from 2 to 12 , but its higher bound is progressively lower. When $q \geq 12$, no positive value of $r$ can be found which satisfies Condition 5 (see online version for colours)

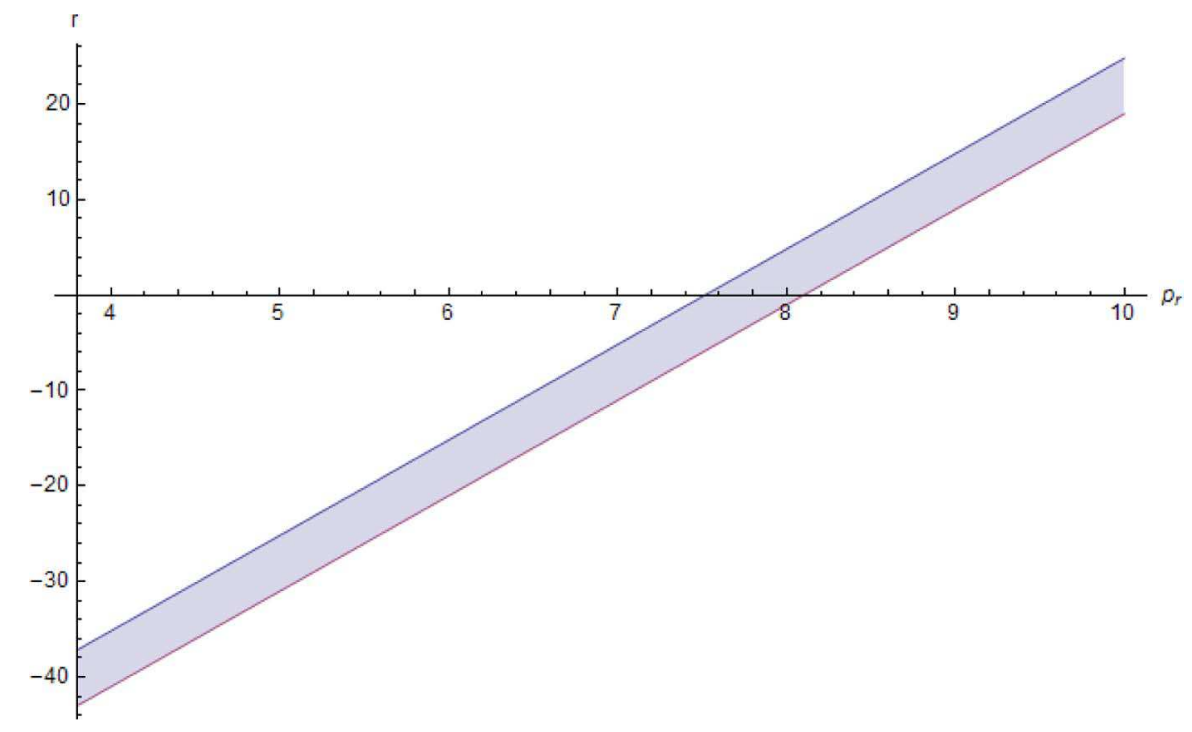

Let

$$
\begin{aligned}
& r s_{H i}(\alpha, \beta, n)=\Psi_{H i}(\alpha, \beta, n) / n+\mu_{X} \alpha, \\
& r h s_{L o}(\alpha, \beta, n)=\Psi_{L o}(\alpha, \beta, n) / n+\mu_{X} \alpha,
\end{aligned}
$$

as the right hand side of the constraint when the function $\Psi$ has been replaced by its high and low bounds. We have $r h s_{H i}(\alpha, \beta, n)=96$ a constant when $\theta$ is set at its high bound. On the other hand, by inspection we see that $38.00<r h s_{L o}(\beta, \alpha, n) \leq 96$ for all tuples $\{\beta, \alpha\}$ which satisfy the conditions of existence of a QFC (see Figure 9).

So, for any value of $\theta$, the right hand side of the constraint is always bounded from below by 38 and from above by 96 . We can now look at the behaviour of the left hand side.

We first set up four functions for the left hand side of the inequalities: two with the upper limit on $r$ for the shipper and carrier and two using the lower limits on $r$ for both players. The lower limit on $r$ comply with the condition of a positive or null fixed fee $r$ so that the MPC is a coordinating contract in the sense established by Cachon and Lariviere (2001) (in the present numerical instance, this low limit is $r=0$ ).

$$
\begin{aligned}
& l h s_{L o}^{s}(q)=r_{L o}(q)+s(q) \mu_{X}(q)+2 \overline{F_{X}}(q)+\varphi^{s}(q) \\
& l h s_{H i}^{s}(q)=r_{H i}(q)+s(q) \mu_{X}(q)+2 \overline{F_{X}}(q)+\varphi^{s}(q) \\
& l h s_{H i}^{c}(q)=r_{H i}(q)+18+(s(q)-1.8) \mu_{X}(q)+\varphi^{c}(q) \\
& l h s_{L o}^{c}(q)=r_{L o}(q)+18+(s(q)-1.8) \mu_{X}(q)+\varphi^{c}(q) .
\end{aligned}
$$

These four functions are represented in Figure 10. The left plot represents the left hand side set of inequalities when $r$ takes the high value (the lower curve plots the carrier's 
constraint and the higher one the shipper's). The right plot represents the carrier's and the shipper's constraints when $r$ takes the lower possible bound.

Figure 9 The right hand side function $r h s_{L o}(\beta, \alpha, n)$ evolves between 38 and 96 for all tuples $\{\alpha, \beta\}$ which satisfy to the existence of a mutually acceptable QFC (see online version for colours)

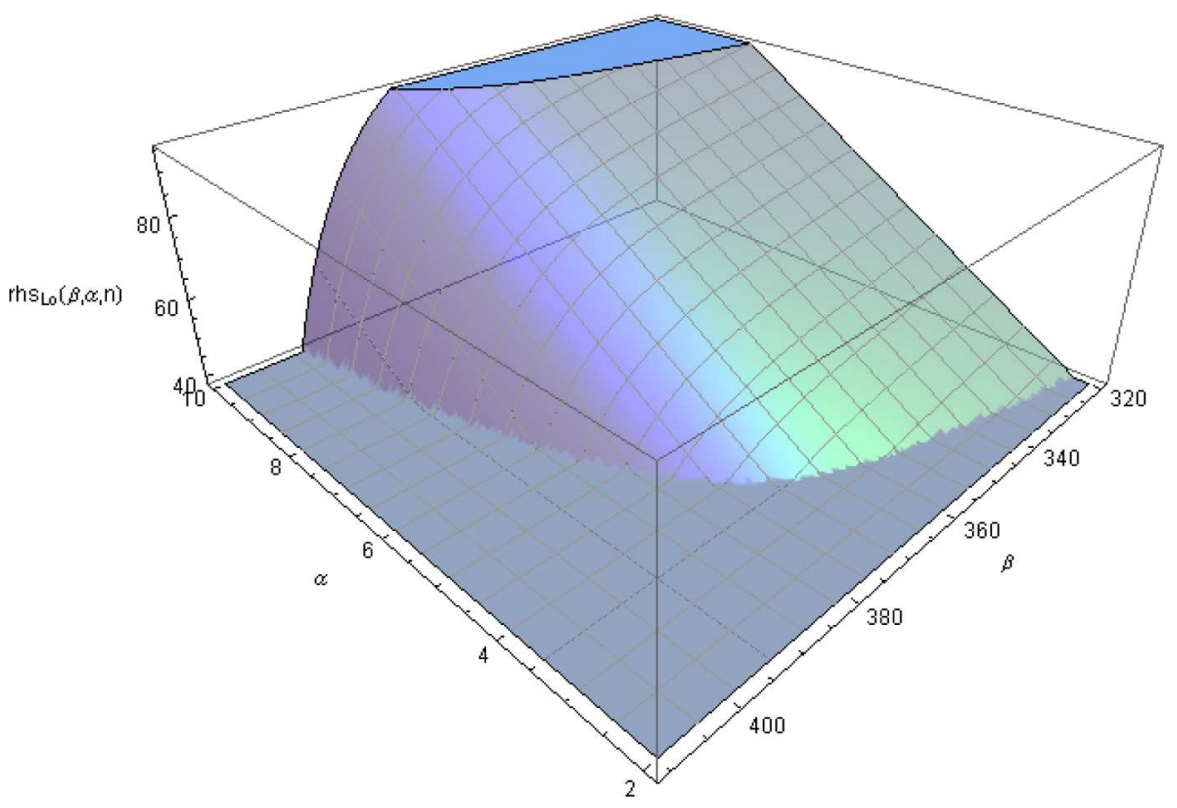

Figure 10 The grey areas are the available domains expressed in money units in which the right hand side of (58) can evolve
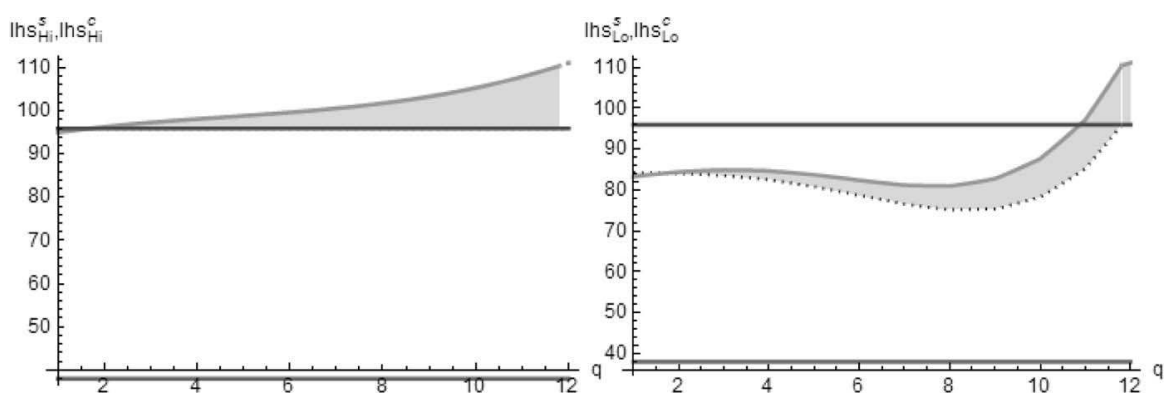

If the MPC dominates strictly the QFC, the following must be verified:

$$
\left\{\begin{array}{l}
l h s_{H i}^{c}(q)>r h s_{H i}(\alpha, \beta, n)>l h s_{H i}^{s}(q) \\
l h s_{L o}^{c}(q)>r h s_{L o}(\alpha, \beta, n)>l h s_{L o}^{s}(q) .
\end{array}\right.
$$

However, what can be observed from the left-hand graph in Figure 10 for the upper limit of $r$ is that $l h s_{H i}^{s}(q)>r h s_{H i}(\alpha, \beta, 40)$ and $l h s_{H i}^{c}(q)=r h s_{H i}(\alpha, \beta, 40)$, 
so that neither contract dominates. Whereas, in the right-hand graph, when $r=0$ and $2 \leq q \leq 11$, inequalities (64) are satisfied: the MPC effectively dominates the QFC. When $q \geq 12$, we see that no contract can be chosen simultaneously by both players because the required conditions spelled out in 7 for a QFC are not satisfied either.

We conclude that a set of parameters of a MPC which dominates the QFC exist which satisfy both players.

The MPC which yields the highest return to the shipper when taken in isolation has $q=8$ and $r=0$, i.e., its low bound. In the same way, the MPC which yields the highest profit to the carrier is when $q=11$. Without knowing the balance of power between both, we have no way of pinpointing the set of parameters of the MPC on which both will agree: $8 \leq q \leq 11$ and $0 \leq r \leq r_{H i}(8)$ (as presented in Figure 5).

\section{$G$ Condition for a contract to dominate both other contracts (Condition 10)}

The conditions for the emergence of a contract over both other contracts means that Condition 10 must be satisfied for one of the three contracts. As seen in the previous subsections, the MPC dominates the PRC when $q \geq 2$ and is preferred to the QFC when $2 \leq q \leq 11$. So, the MPC is preferred overall and no other contract can be preferred in the present numeric instance over 40 periods.

In the unlikely case where a commitment of $q=1$ is preferred, then both the PRC and QFC come back into play as possible choices. Either can be retained because sufficient domains exist for the economic result for each player to be equal. Note however that the conditions are tighter than when both are compared on their own merits without comparing them to the MPC: we need to have $p_{r} \geq 8.425$ as observed in $\mathrm{E}$.

\section{F Numeric example with fixed spot price}

In this instance, we consider that the spot market price for transport does not fluctuate. Due to the fact that the only random variable now is demand, the functions $\varphi()$ in (9), $s(q)$ in (15) change and we can write, adding a superscript 1 to indicate that they represent a univariate state of the world and naming $P$ the fixed spot price,

$$
\begin{aligned}
\varphi^{s 1}(q)= & \frac{1}{\overline{F_{X}}(q)} \int_{q}^{Q_{H i}}(x-q) P f_{X}(x) \mathrm{d} x \\
\varphi^{c 1}(q)= & \frac{1}{F_{X}(q)} \int_{0}^{q}(q-x)(P-c) f_{X}(x) \mathrm{d} x \\
& +\frac{1}{\overline{F_{X}}(q)} \int_{q}^{Q_{H i}}(x-q)(P-c) f_{X}(x) \mathrm{d} x \\
s^{1}(q)= & \frac{I f_{X}(q)-\varphi^{s 1^{\prime}}(q)}{1-F_{X}(q)} .
\end{aligned}
$$

Regarding the functions which intervene in the calculations of the QFC, the definitions of functions $g_{1}(\beta, n)$ and $g_{2}(\beta, n)$ are the same in both the bivariate and univariate cases. So, we also have the same definition for $\Psi(\beta, \theta, n)$. 


\section{A Condition of existence of a MPC in the univariate case}

We first want to see what conditions for the parameters of the MPC would make this contract eligible. From Condition 3, when adapted to the case at hand, we can present the constraints on $r$ in the Figure 11. Since, to be a coordinating contract, we need that the parameter $r$ be positive, the resulting possible tuples $\{q, r\}$ are restricted to the grey vertical bars in that figure.

Figure 11 Fixed spot price: the parameter $r$ in terms of $q$, the segments are the tuples $\{q, r\}$ that enable the MPC to coordinate the players

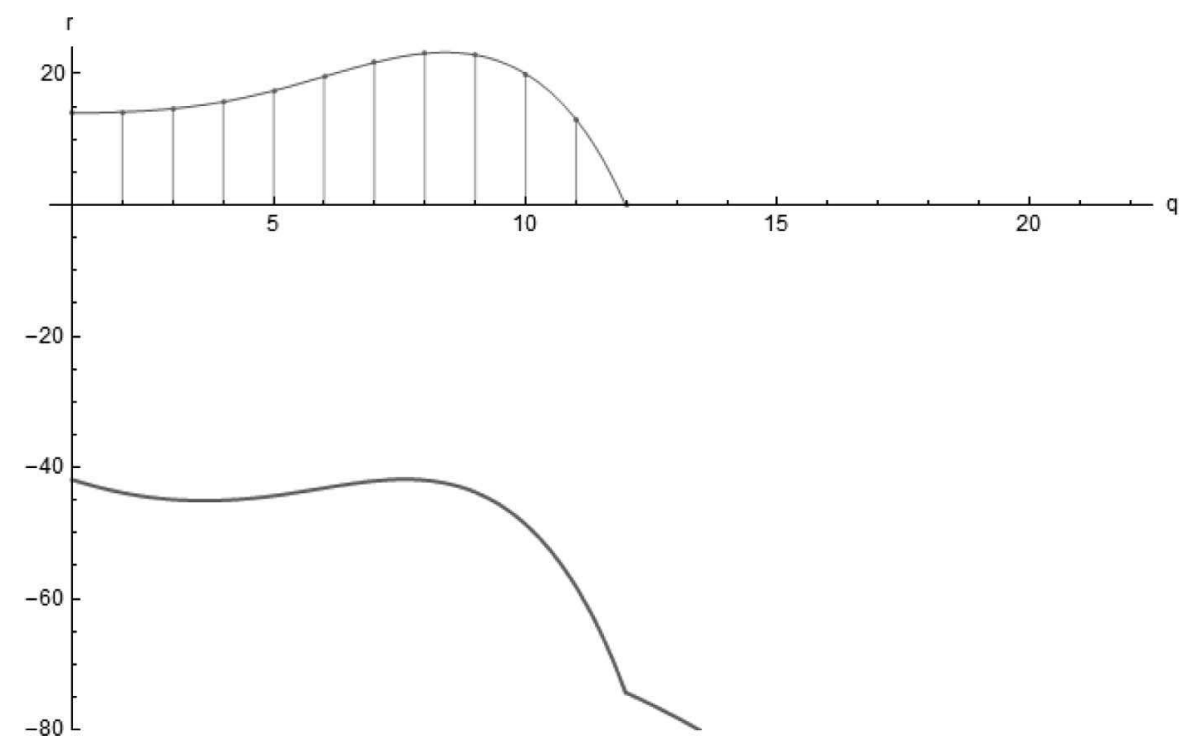

The corresponding objective functions of the players can be presented in the Figure 12. Because the fixed fee is at least equal to 0 and cannot take negative values, the shipper makes the most of her budget by choosing $q=8$. If we had left $r$ take negative values, the shipper would have had an increasing objective function in terms of $q$. As the carrier is also motivated by a high commitment, they would have settled for a commitment as high as possible (like $q=22$, or 4 standard deviations above the expected demand): a contract which is clearly not a coordinating one as the shipper would fail to fill the capacity put at her disposition, negating the benefit of this type of contract.

\section{$B$ Condition of existence of a QFC in the univariate case}

We now turn to the QFC. The participation constraints from Condition 4 on $\theta$ are the same as in the bivariate case.

The envelopes in the volume $\{\alpha, \beta, \theta\}$ which represent the boundaries of existence of the penalty $\theta$ are also the same as the ones presented in Section $\mathrm{C}$ in Figure 6. The vectors of values which describe this volume constitute the mutually agreeable sets of parameters for a QFC to be eligible in the univariate case also.

We now compare the MPC and the PRC. 
Figure 12 Fixed spot price: objective functions of shipper and carrier: the objective functions show that both players have a weak incentive to extend the commitment to its highest possible level
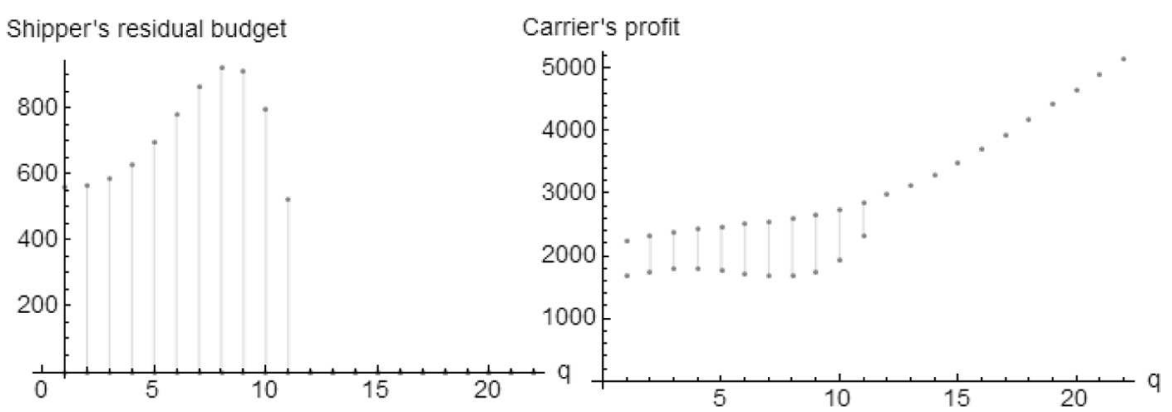

\section{Condition of dominance of a MPC over a PRC}

Which parameters of the MPC would make it superior to the PRC for both players to adopt it? From Condition 5 adapted to the univariate case, we have

$$
\begin{aligned}
& r \leq 10 p_{r}-2 \overline{F_{X}}(q)-\varphi^{s 1}(q)-s^{1}(q) \mu_{X}(q) \\
& r \geq 10 p_{r}-\varphi^{c 1}(q)-1.8\left(10-\mu_{X}(q)\right)-s^{1}(q) \mu_{X}(q) .
\end{aligned}
$$

We present in Figure 13 the resulting envelopes. We see that the lower bound (topmost envelope in the figure) crosses the higher one when $q=1.985$, which means that the conditions for the dominance of a MPC can only be fulfilled when $2 \leq q \leq 12$. For the PRC to dominate, we have to satisfy 23 and this can only be the case when $q=1$ (not represented in the graph in Figure 13). In other cases, either $r$ would be negative or $p_{r}$ would have to exceed 9.6, the shipper's participation constraint. Within those limits, the MPC dominates the PRC for both players. The PRC is preferred when $q=1$, as the inequalities 23 in Condition 5 would be satisfied. Such solutions in terms of $r$ in terms of $p_{r}$ are presented as the shaded area in Figure 14.

\section{$D$ Comparing the QFC and PRC in the univariate case}

Since Condition 6 which presents the cases of dominance of one contract over the other can be applied to the univariate case in the same way as in the bivariate one, and by replacing $\theta$ by its upper and lower bounds (expressed in terms of $\alpha$ and $\beta$ ) in the definition of function $\Psi_{H i}(\beta, \theta, n)$, we obtain a set of constraints on $p_{r}$ in Condition 1 which can be written in the following way

$$
\left\{\begin{array}{l}
3.8 \leq \alpha+\frac{\Psi_{H i}(\alpha, \beta, n)}{n \mu_{X}} \leq 9.6 \\
3.8 \leq \alpha+\frac{\Psi_{L o}(\alpha, \beta, n)}{n \mu_{X}} \leq 9.6 .
\end{array}\right.
$$

If these inequalities were not true, the QFC and PRC would not be equivalent, so none could be chosen. 
Figure 13 Fixed spot price: the available values of $r$ lie between both envelopes. Not all values of $p_{r}$ are compatible (see online version for colours)

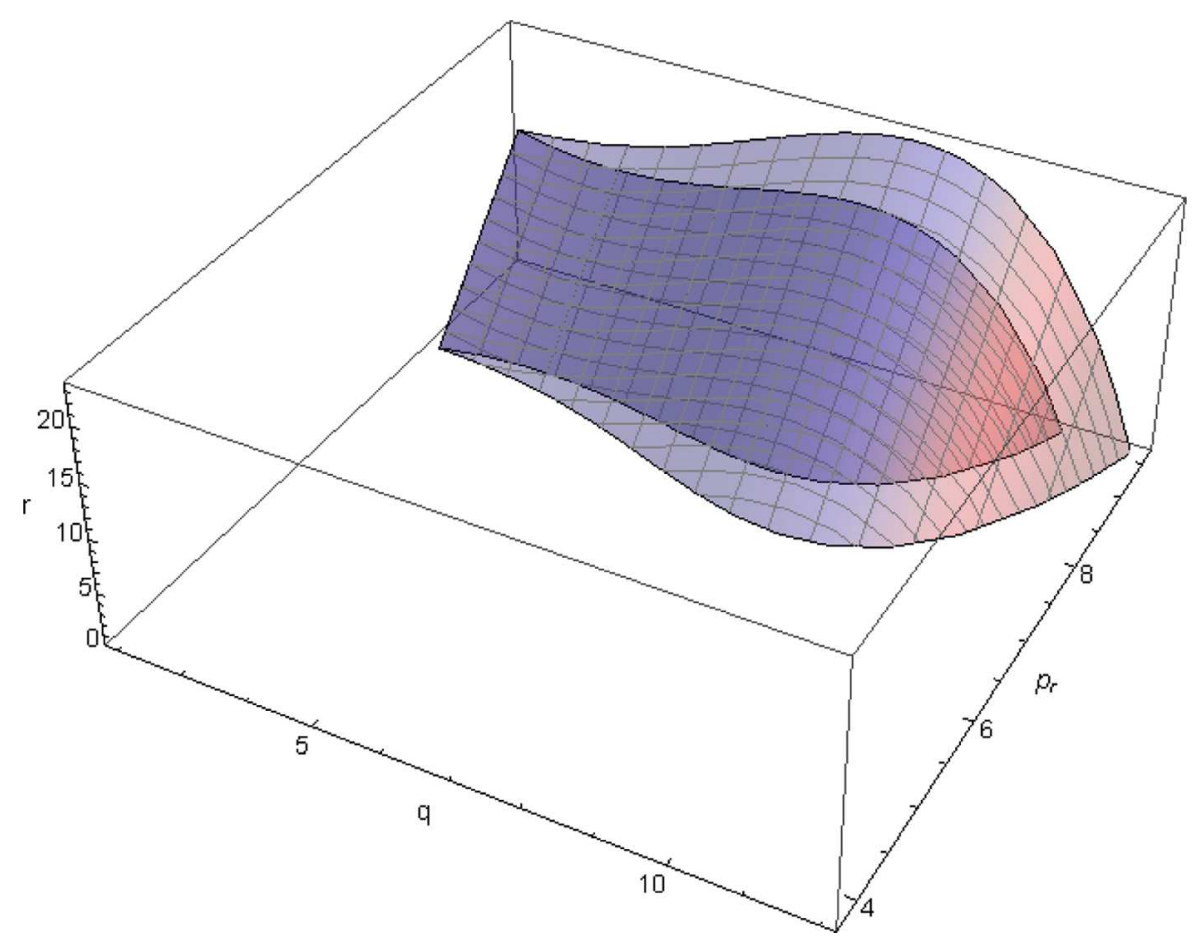

Figure 14 Fixed spot price: the shaded area represents the available values of $r$ given $p_{r}$ for a PRC to dominate a MPC, when $q=1$

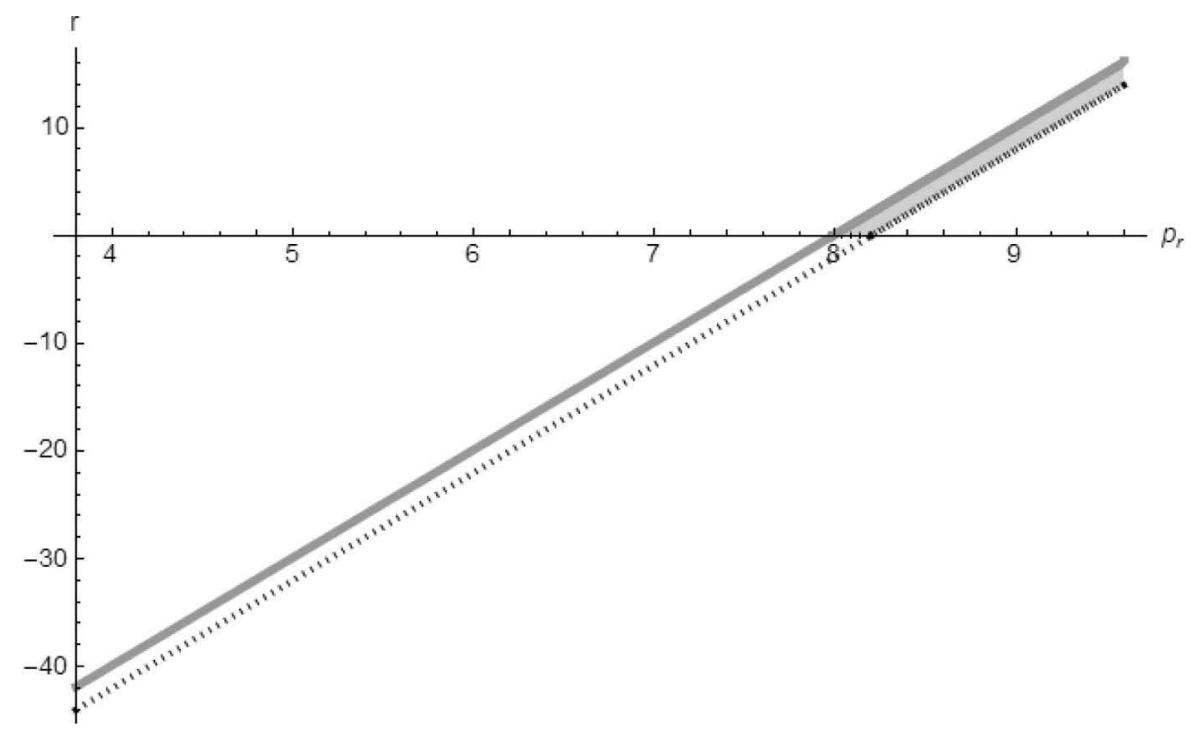


When one plots the expressions $\alpha+\frac{\Psi_{H i}(\alpha, \beta, n)}{n \mu_{X}}$ and $\alpha+\frac{\Psi_{L o}(\alpha, \beta, n)}{n \mu_{X}}$, one sees that the first has a minimum of 3.8 and a maximum of 9.6, except for those tuples $\{\alpha, \beta\}$ which in any case do not satisfy the participation constraints of the shipper. In the same way, the second expression has a minimum of 9.6 except for those tuples in $\{\alpha, \beta\}$ which do not satisfy the participation constraints of the shipper. The possible tuples $\{\alpha, \beta\}$ which satisfy all requirements for equivalence between either contract are represented as the shaded area in Figure 15.

Figure 15 The shaded surface represents the possible tuples $\{\alpha, \beta\}$ for which a QFC and a PRC can be equivalent. The penalty $\theta$ can take any of the possible values that are allowed under Condition 4 (see online version for colours)

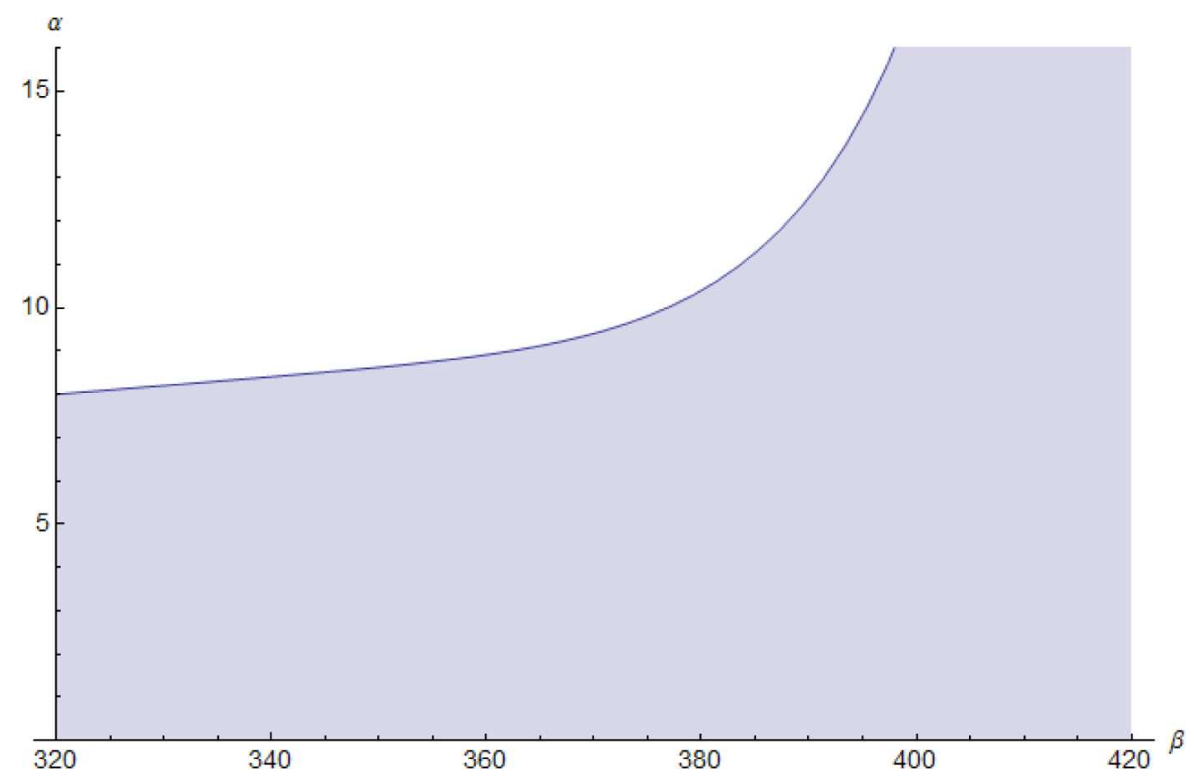

\section{E Condition of dominance of a $Q F C$ over a MPC}

Let us evaluate the conditions in which the QFC dominates the MPC. Adapting Condition 7 from the bivariate case by replacing functions $\varphi^{s}(q)$ and $\varphi^{c}(q)$ by their equivalents in the univariate case, we correspondingly change the functions presented in (60) and rename them with an added superscript 1: lh $s_{H i}^{c 1}, l h s_{H i}^{s 1}, l h s_{L o}^{c 1}$ and $l h s_{L o}^{c 1}$.

As in the bivariate case, the right hand side of the inequalities are the same as in the bivariate instance.

The functions lhs describe the segments within which $r$ can be set in terms of $q$, as can be seen in Figure 16: the left hand graph represents the high and low bounds when $r$ is at its high bound, the right hand graph represents the same high and low limits taken by $l h s_{L o}^{i}(q), i \in\{s, c\}$ when $r$ is at its low bound (in the present case: $r=0$ ). The higher and lower limits of $r h s(\alpha, \beta, n)$ are also presented.

We have to solve

$$
\left\{\begin{array}{l}
\operatorname{lh} s_{H i}^{c 1}(q)<r h s_{H i}(\alpha, \beta, n)<l h s_{H i}^{s 1}(q) \\
\operatorname{lh} s_{L o}^{c 1}(q)<r h s_{L o}(\alpha, \beta, n)<l h s_{L o}^{s 1}(q) .
\end{array}\right.
$$


Figure 16 The left hand side of inequalities in (60) suitably adapted to the univariate case are presented together with the upper and lower limits on $\operatorname{rhs}(\alpha, \beta, n)$ : left-hand graph when $r$ is at its highest, right-hand graph, when $r=0$
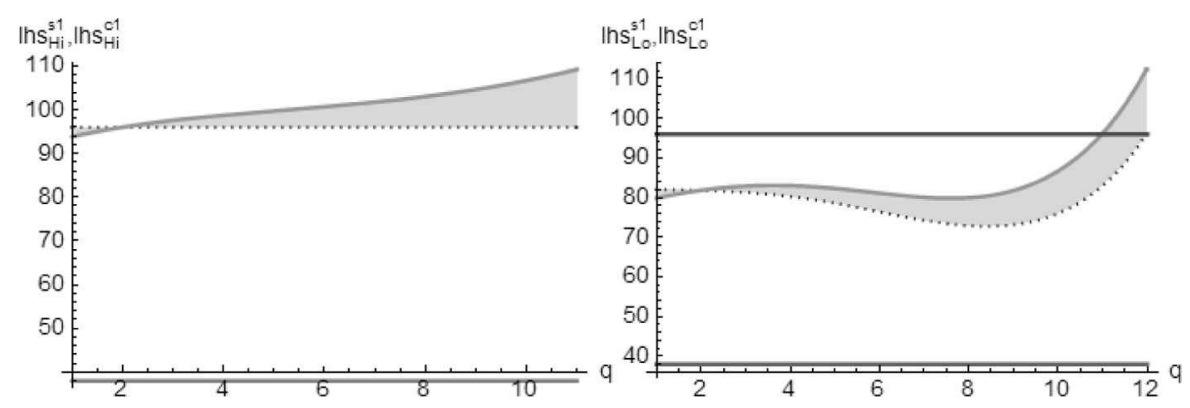

By inspection, $l h s_{L o}^{c 1}>l h s_{L o}^{s 1}$ and $l h s_{H i}^{c 1}>l h s_{H i}^{s 1}, \forall q \geq 2$. We also observe that in two other situations: (a) when $q \geq 12$, $l h s_{L O}^{c 1}>r h s_{H i}$ and (b) when $r$ takes its higher bound and $q \geq 2, l h s_{H i}^{s 1}=r h s_{H i}$ and $l h s_{H i}^{c 1}>r h s_{H i}$, the players would choose different contracts. If there are values of $\alpha, \theta$ and $\beta$ which enable the inequalities 31 in Condition 7 to be satisfied, then the MPC dominates the QFC and if no values of $\{\alpha, \beta, \theta\}$ can be found neither contract can be chosen simultaneously.

To describe here all the possible solutions of contracts QFC and MPC which fall in this category would be fastidious. We present below the solution for one solution for the MPC and corresponding solution set and method to evaluate it.

When $q=8, r=0, s=5.983$, we have $l h s_{L o}^{c 1}(8)=79.9489$ and $l h s_{L o}^{s 1}(8)=72.909$. Which suitable sets of $\{\alpha, \beta, \theta\}$ allow $72.909<\Psi(\beta, \theta, 40) / 40+\mu_{x} \alpha$ ? For example, as can be gathered from Figure 17, once $\{\alpha, \beta\}$ have been determined, the available penalties are the ones for which $72.909<\Psi(\beta, \theta, 40)+\mu_{x} \alpha<79.9489$, so for example, if $\alpha=5, \beta=380$, then $0<\theta<0.9663$.

\section{F Condition of dominance of a PRC over a MPC and equivalent to a QFC}

As seen above for the preeminence of the PRC over the MPC, this means that we need $q=1$ for a PRC to dominate. If this is so, the required values of $p_{r}$ are as presented in Figure 14, i.e., $8.20<p_{r} \leq 9.6$.

Since we also need an equivalent QFC, we must have Condition 6 satisfied. This is true when

$$
\left\{\begin{array}{l}
\theta<\left[3840-n \mu_{X} \alpha-\mu_{P}\left(g_{2}(\beta, n)-\frac{\beta}{\overline{F_{Y_{n}}}(\beta)}\right)\right] \frac{1}{\frac{\beta}{F_{Y_{n}}(\beta)}-g_{1}(\beta)} \\
\theta>\left[3280-n \mu_{X} \alpha-\mu_{P}\left(g_{2}(\beta)-\frac{\beta}{\overline{F_{Y_{n}}}(\beta)}\right)\right] \frac{1}{\frac{\beta}{F_{Y_{n}}(\beta)}-g_{1}(\beta)}
\end{array}\right.
$$

This set of conditions is reminiscent of the ones encountered in E. The lower and upper limits to the possible values of $\theta$ are very close. As an example, when $\alpha=5, \beta=405$ and $n=40$, we have $21.62<\theta<23.60$. The set of available QFC parameters $\{\alpha, \beta, \theta\}$ are presented in Figure 18. 
Figure 17 Fixed spot price: the upper and lower bounds of $r h s^{1}(\alpha, \beta, 40)$ are 72.909 and 79.9489. The graph represents a volume containing the possible tuples $\{\alpha, \beta\}$ when $\theta$ is at its low bound: the point $\{5,380\}$ is in the solution set, $\{8,340\}$ is not

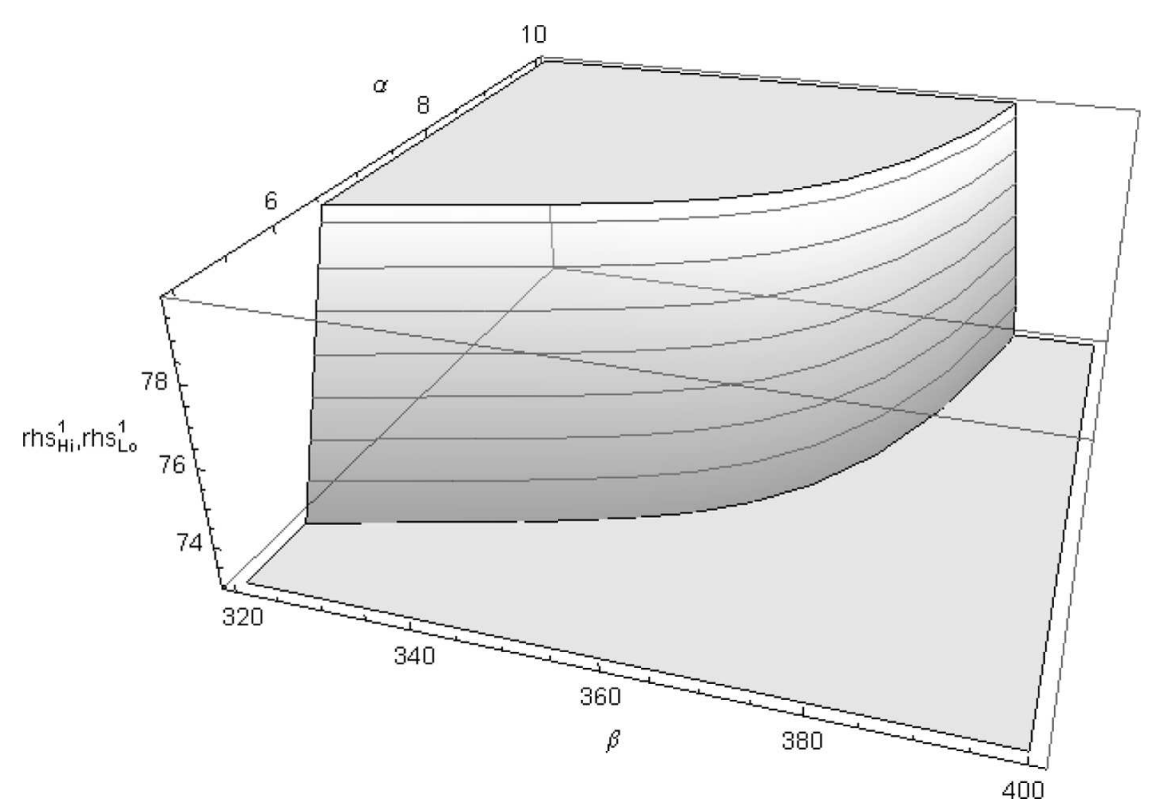

Figure 18 Fixed spot price: the figure represents two envelopes which nearly coincide: the lower and upper limits of the value of $\theta$ in terms of the tuples $\{\alpha, \beta\}$ solution to (68) and which describe the QFC which is equivalent to the dominant PRC

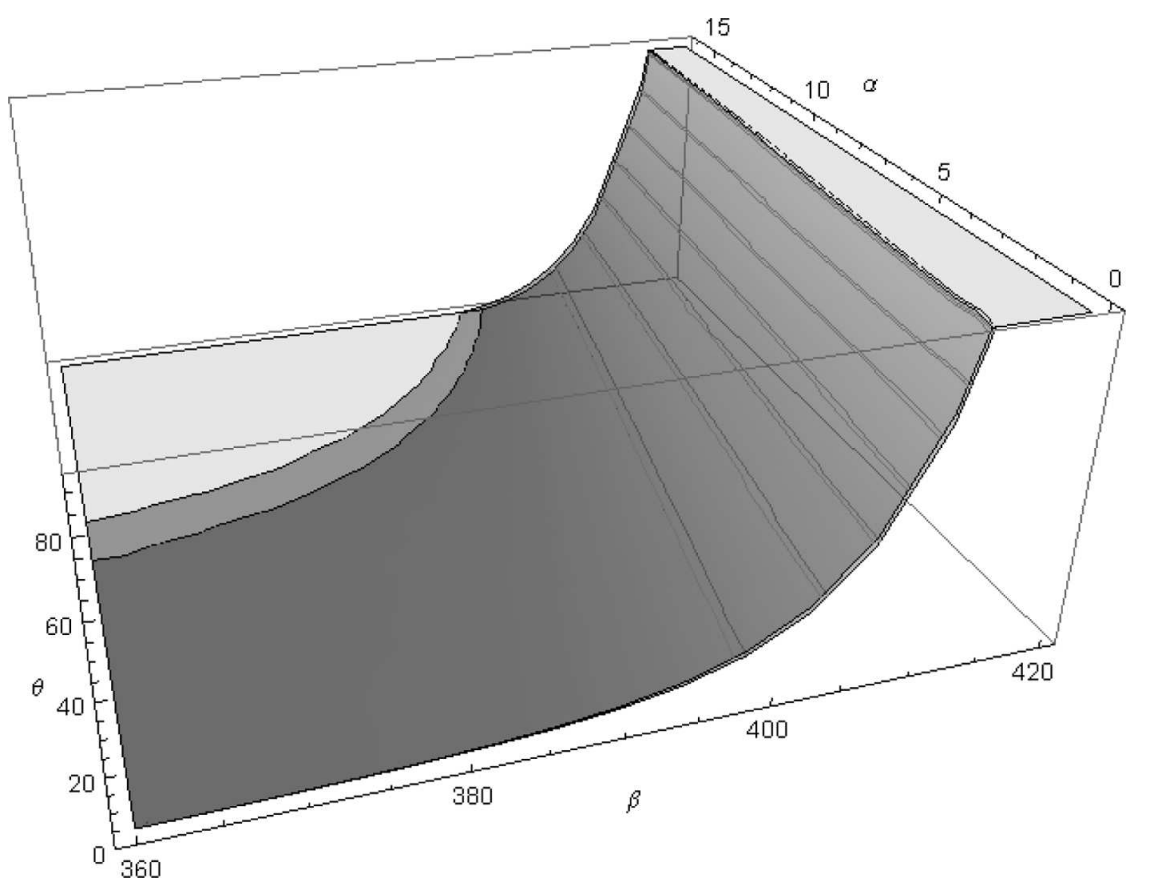




\section{$G$ Condition of dominance of a MPC over both a QFC and a PRC}

Since we already have established the conditions for a MPC to dominate both a PRC and a QFC separately, we just have to ensure that the PRC and QFC are equivalent to obtain the necessary and sufficient conditions for a MPC to dominate both others.

We have seen earlier that the only remaining commitment $q$ which enables the satisfaction of theses conditions is when $2 \leq q<12$, and the parameters of the QFC allow it to be equivalent to the PRC. Further, the inequality set (67) has to be satisfied.

We represent in Figure 19 one set of tuples which enable a QFC to exist and be comparable to a MPC when $q=8$. It has been chosen after setting $\theta$ at its low boundary. A similar graph could have been drawn for other values of $\theta$ and $q$.

Figure 19 Fixed spot price: the figure represents the lower and higher limits of the tuples $\{\alpha, \beta\}$ which enable the right hand side of the inequalities in (31) to be between $l h s_{L o}^{c 1}$ and $l h s_{L o}^{s 1}$ and hence a compatible QFC to exist. The case where $q=8$ is represented, the other values of $q$ would be similar

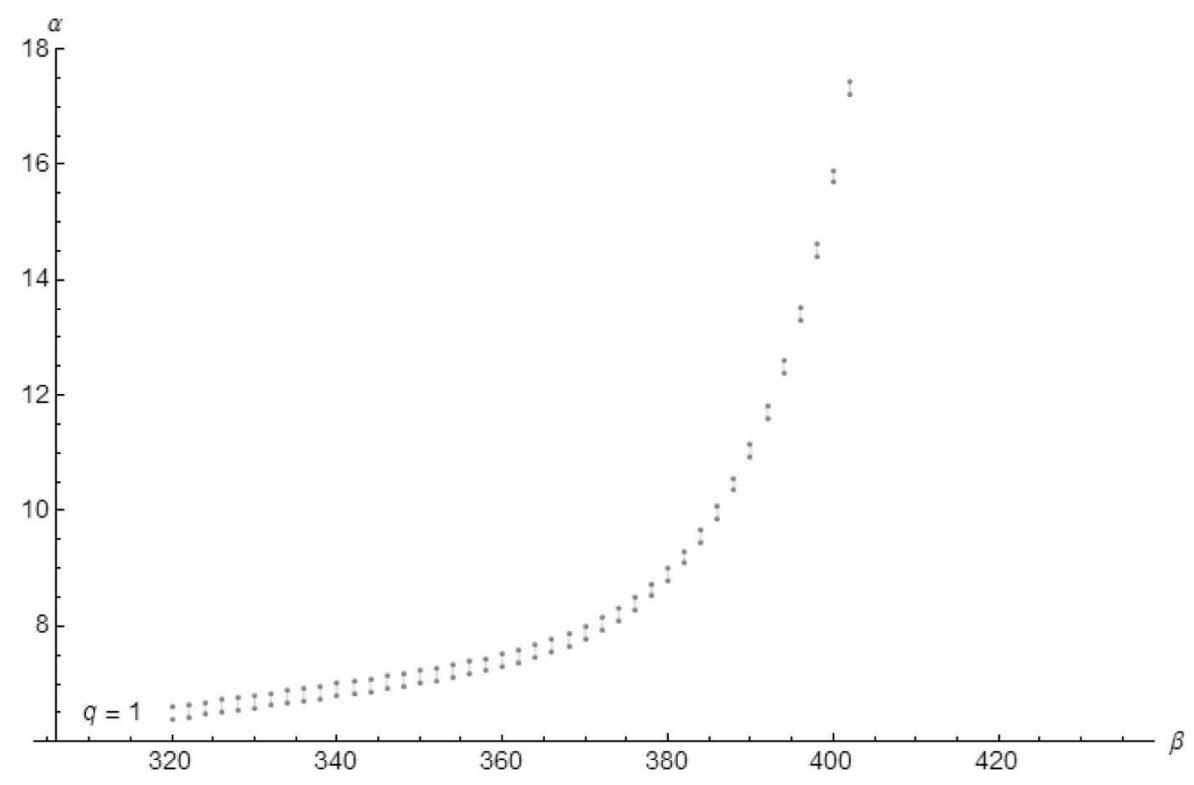

$H$ Condition of dominance of a QFC over both a MPC and a PRC

We have seen earlier that the set of values which enable a QFC to be chosen preferentially require that the MPC be first dominated; this means that $q=1$, further the penalty must satisfy the inequality set (68).

This leads to profit functions which are plotted in Figure 20: the result is independent of the commitment as both $\alpha$ and $\theta$ are in fact the coordinating factors. This graph leads us to believe that the variance of demand must have a high impact on the final result both for the shipper and carrier when a high commitment $\beta$ is agreed upon: if the realised demand does not meet the expected one, the result must be very different from the ones plotted here given that both $\alpha$ and $\theta$ are relatively expensive (when $\beta=436, \alpha=300.3$ and $7.128<\theta<18.27$ ). 
Figure 20 Fixed spot price: the figure represents the lower and higher limits of the objective functions of the shipper and carrier in the case that the dominated MPC has $q=1$ for all capacity commitments

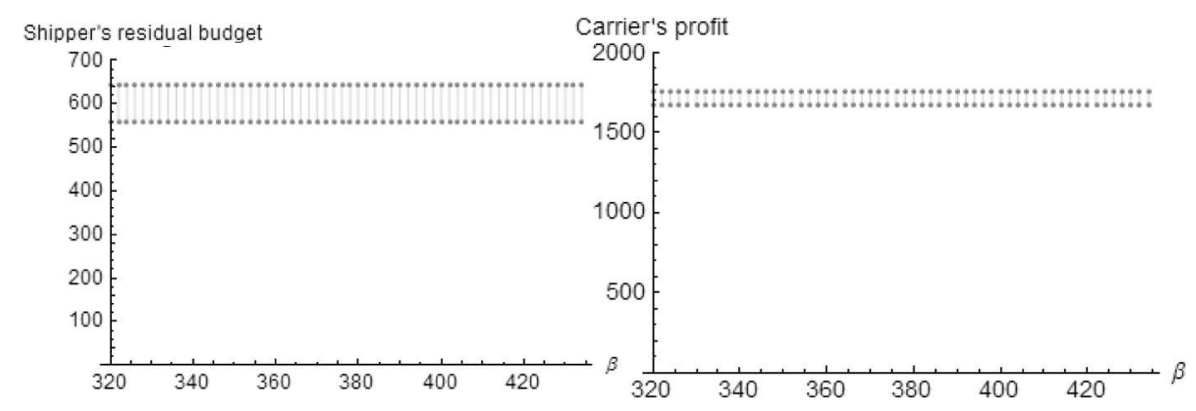

\section{G Variance of results for all strategies}

We proceed to evaluate the variance of each contract. We start with the variance of the profit or retained budget for carrier and shipper in the case where they both rely in the spot market for their needs.

\section{A Variance of residual budget to the shipper when buying from the spot market}

The expected cost of going to the spot market every period to buy the required capacity to the shipper is the result of the product of the two random variables $X$ and $P$, demand and spot market price. By definition,

$$
E(X Y)=\operatorname{Cov}(X, P)+\mu_{X} \mu_{P}=82.25 .
$$

Hence, the expected residual budget is over 40 periods:

$$
\Pi_{\text {spot }}=B(40)-40(2+82.25)=470 .
$$

The variance is by definition over one period

$$
\operatorname{Var}(X P)=E\left(X^{2} P^{2}\right)-E(X P)^{2}=1186.3 .
$$

If we consider that the number of periods is independent of demand, the variance of a procurement strategy using only the spot market over 40 periods is 40 times the preceding result:

$$
V_{\mathrm{spot}}=47451 .
$$

Let us now evaluate the variance for the contracts.

\section{$B$ Variance of the cost of the PRC}

The only risk affecting the shipper in this contract is the demand risk, hence the variance of the cost to her of using this contract is reduced to the variance of demand times the number of periods and the square of the per unit price:

$$
\operatorname{Var}_{P R C}\left(p_{r}\right)=n p_{r}^{2} \sigma_{X}^{2}
$$


In our bivariate numerical instance this can be represented in terms of $p_{r}$ in Figure 21. As can be seen, $5198<\operatorname{Var}_{P R C}<33178$.

Figure 21 Variance of a PRC according to $p_{r}$ over 40 periods (see online version for colours)

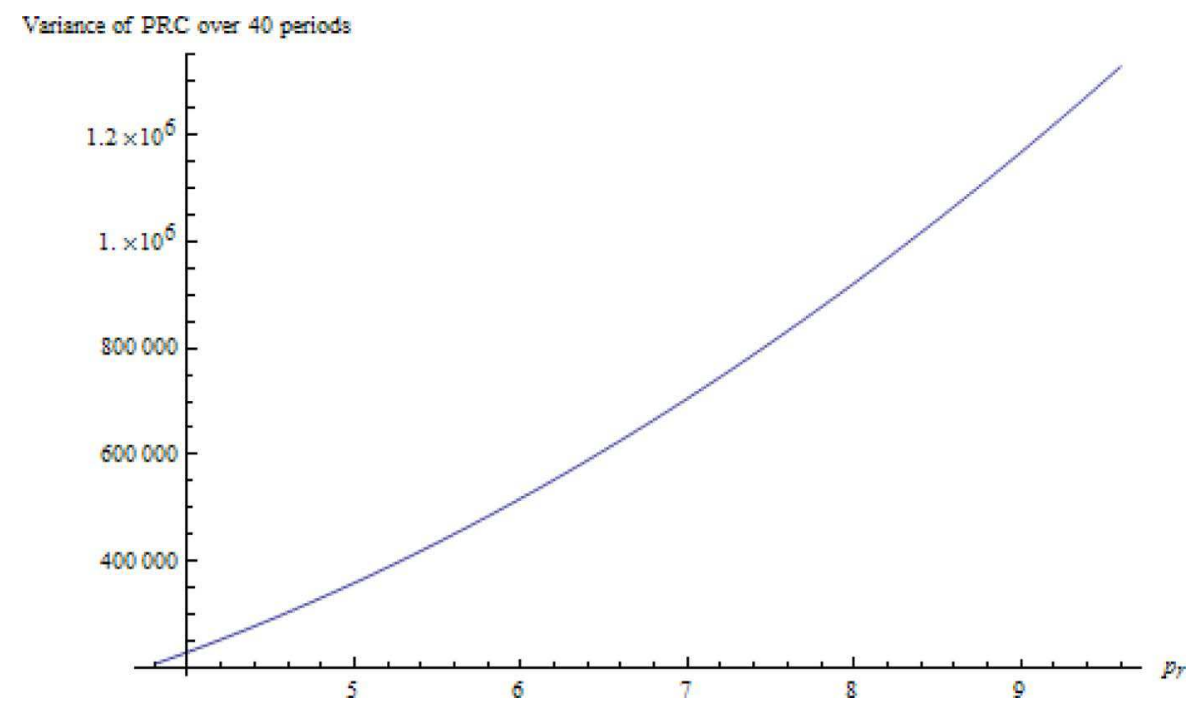

\section{$C$ Variance of the cost to the shipper of the MPC}

Since the contract does not use the spot market when demand is less than $q$, we can distinguish, for the purpose of evaluating the covariance, between the case when demand is less than $q$ and when it is higher. Let us define

$$
\begin{aligned}
& v(q)=\operatorname{Var}(X \mid X<q) \\
& z(q)=\operatorname{Var}((X-q) P \mid X>q) .
\end{aligned}
$$

We can write the conditional variance of the demand when it is less than the committed capacity as

$$
\begin{aligned}
v(q) & =E\left(X^{2} \mid X<q\right)-E(X \mid X<q)^{2} \\
& =\frac{1}{F_{X}(q)} \int_{0}^{q} x^{2} f_{X}(x) \mathrm{d} x-\frac{1}{F_{X}(q)^{2}}\left(\int_{0}^{q} x f_{X}(x) \mathrm{d} x\right)^{2} .
\end{aligned}
$$

However, the covariance of demand and spot market price when demand exceeds the committed capacity must be written

$$
\begin{aligned}
z(q) & =E\left([(X-q) P]^{2} \mid X>q\right)-E((X-q) P \mid X>q)^{2} \\
& =e_{X}(q)-q_{X}(q)^{2}
\end{aligned}
$$

with

$$
e_{X}(q)=\frac{1}{1-F_{X}(q)} \int_{v}^{P_{H i}} \int_{q}^{Q_{H i}}(x-q)^{2} y^{2} f(x, y) \mathrm{d} x \mathrm{~d} y
$$




$$
q_{X}(q)=\frac{1}{1-F_{X}(q)} \int_{v}^{P_{H i}} \int_{q}^{Q_{H i}}(x-q) y f(x, y) \mathrm{d} x \mathrm{~d} y .
$$

The total variance of the MPC to the shipper is the sum of both variances since they occur in distinct domains:

$$
\operatorname{Var}\left(V_{2}\right)=s(q)^{2} v(q)+z(q) .
$$

So total variance of the MPC decreases to a minimum when the capacity commitment equals expected demand as can be seen in Figure 22 before shooting up again as the commitment encompasses the expected demand plus several standard deviations. At the minimum, it is lower than the variance of the spot market procurement (topmost line).

Figure 22 Variance of a MPC and spot market buying strategy according to $q$ when taken in isolation (see online version for colours)

Variance of MPC and Spot

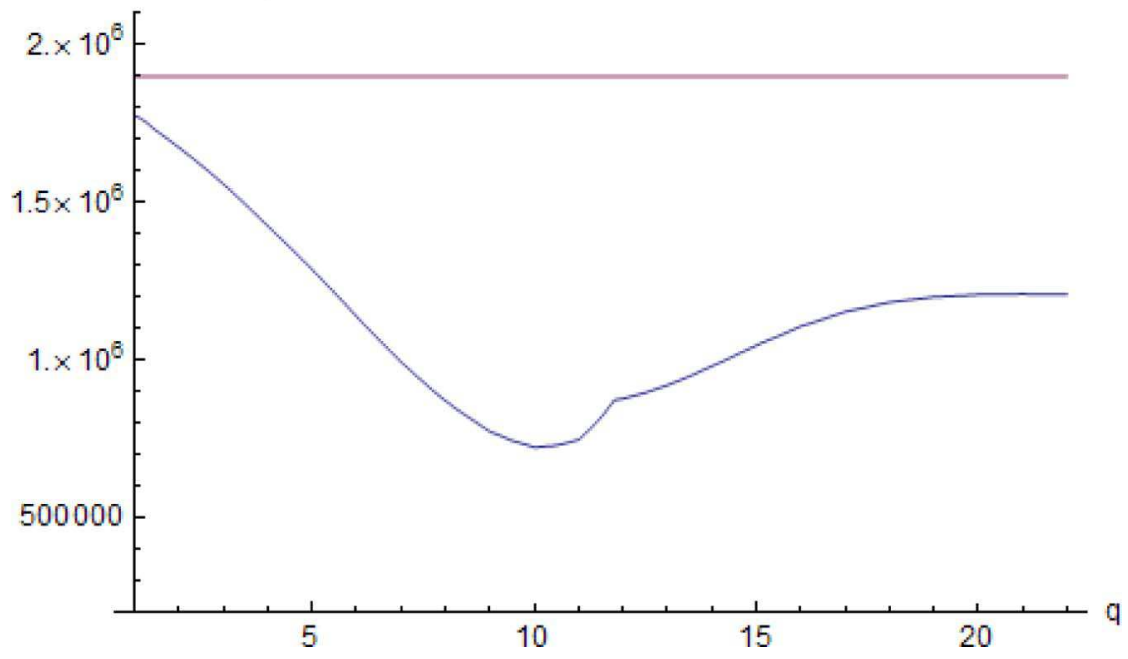

$D$ Variance of the cost of the QFC to the shipper

We first need the variance of the sum of demands when they sum less than the commitment $\beta$ :

$$
\begin{aligned}
V_{g_{1}}(\beta, n) & =E\left(X^{2} \mid \sum_{i=1}^{n} x_{i} \leq \beta\right)-E\left(X \mid \sum_{i=1}^{n} x_{i} \leq \beta\right)^{2} \\
& =\frac{1}{F_{Y_{n}}(\beta)} \int_{0}^{\beta} u^{2} f_{Y_{n}}(u) \mathrm{d} u-\frac{1}{F_{Y_{n}}(\beta)^{2}}\left[\int_{0}^{\beta} u f_{Y_{n}}(u) \mathrm{d} u\right]^{2} .
\end{aligned}
$$

When the sum of demands is higher than commitment $\beta$, the spot price comes into play and its variance has to be taken into account. The variance is that of a product of random variables, which is $\operatorname{Var}(X Y)=\mathbb{E}\left(X^{2} Y^{2}\right)-\mathbb{E}^{2}(X Y)$. We also know that 
$\mathbb{E}\left(X^{2} Y^{2}\right)=\mathbb{E}\left(X^{2}\right) \mathbb{E}\left(Y^{2}\right)+2 \mathbb{E}^{2}(X Y)$. Hence, the variance of the price for transport when the sum of demands exceeds $\beta$ is written as

$$
\begin{aligned}
\operatorname{Var}\left(P\left(\sum_{i=1}^{n} X_{i}-\beta\right) \mid \sum_{i=1}^{n}>\beta\right)= & \mathbb{E}\left(P^{2}\right) \mathbb{E}\left(\left(Y_{n}-\beta\right)^{2} \mid Y_{n}>\beta\right) \\
& +\mathbb{E}^{2}\left(P\left(Y_{n}-\beta\right) \mid Y_{n}>\beta\right) .
\end{aligned}
$$

So we can write

$$
\begin{aligned}
V_{g_{2}}(\beta, n)= & \frac{1}{\overline{F_{Y_{n}}}(\beta)} \int_{\beta}^{Q Y_{H i}}(u-\beta)^{2} f_{Y_{n}}(u) \mathrm{d} u \\
& +\left[\frac{1}{\overline{F_{Y_{n}}}(\beta)} \int_{\beta}^{Q Y_{H i}} 8(u-\beta) f_{Y_{n}}(u) \mathrm{d} u\right]^{2},
\end{aligned}
$$

naming $V_{g_{1}}$ and $V_{g_{2}}$ the functions of the variance when demand is lower and higher than $\beta$ respectively.

The variance of the residual budget of the shipper with this contract can be resumed to

$$
\operatorname{Var}\left(V_{3}(\alpha, \beta, n)\right)=3^{2} \alpha^{2} n^{2}+\theta^{2} V_{g_{1}}(\beta, n)+\mu_{P}^{2} V_{g_{2}}(\beta, n) .
$$

We present in Figure 23 the resulting variance when $\theta=0.1$, two values of $\alpha=0.1$ and $\alpha=4$ and $\beta$ evolves between 320 and 480. When the penalty increases it induces higher volatility if it surpasses certain levels. At high levels, the volatility in terms of $\beta$ goes through a minimum before increasing again as can be seen in Figure 24 . For example, when $\theta=25$, a minimum of volatility of $0.117 \times 10^{6}$ is achieved with $\beta=395$.

Figure 23 Variance of a QFC according to $\beta$, with $n=40, \theta=0.1, \alpha=0.1$ (lower curve), and $\alpha=4$ (higher curve) when taken in isolation (see online version for colours)

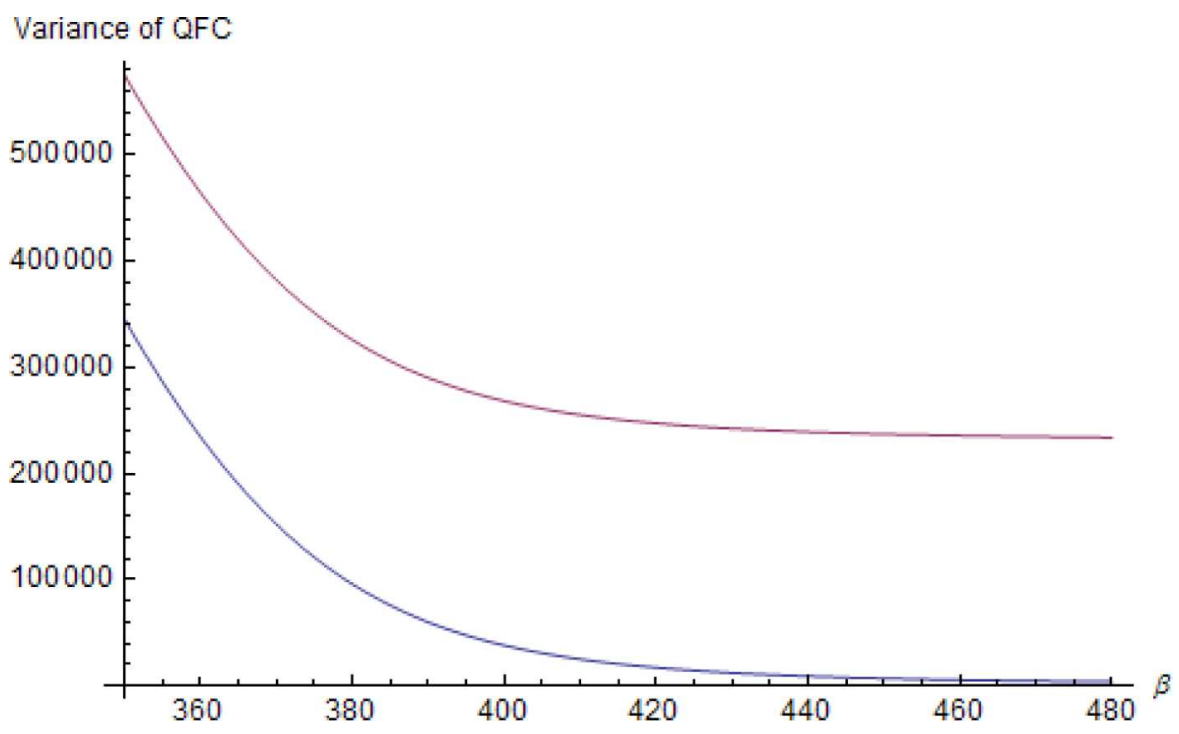


Figure 24 Variance of a QFC according to $\beta$, with $n=40, \alpha=0.1$ and $\theta=1$ (lower curve), and $\theta=15$ (higher curve) when taken in isolation (see online version for colours)

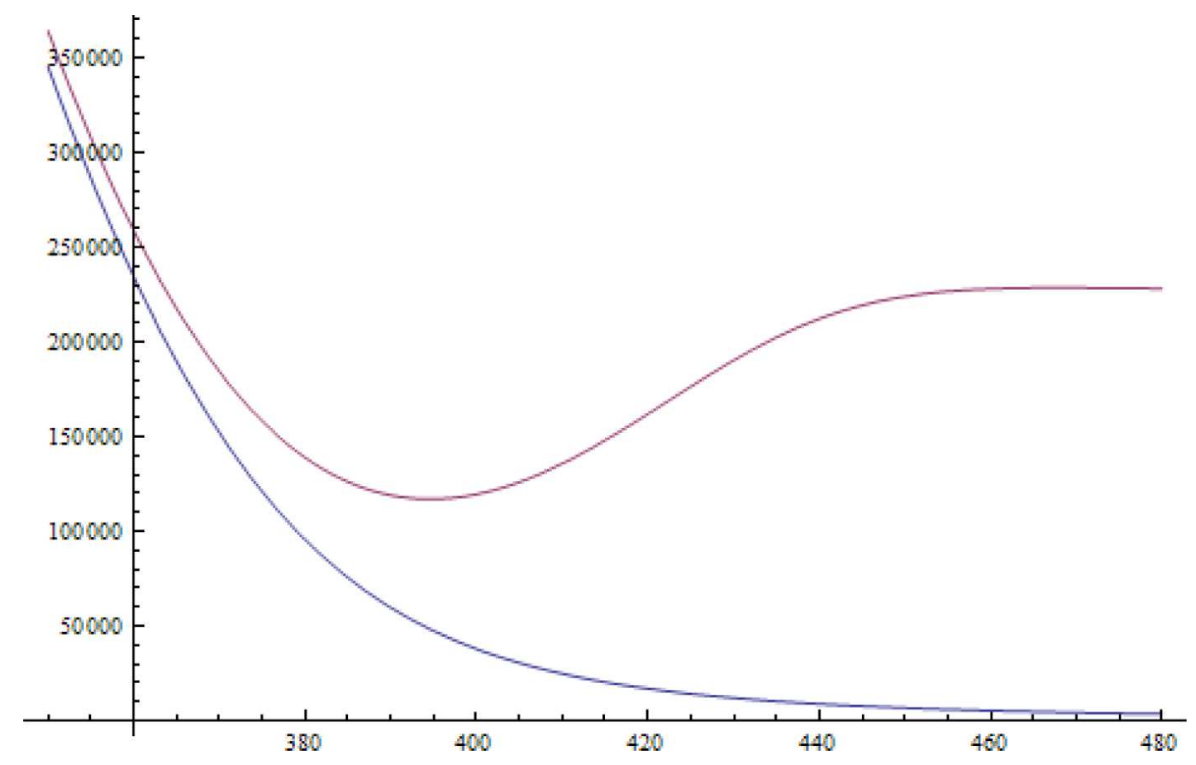

The variance of such a contract is clearly lower than the ones resulting from the use of either the MPC or pure spot procurement.

As a final comparison of results, Table 5 presents the lowest possible variance for each form of transport procurement given the same distribution of demand and spot market prices.

Table 5 Lowest variances for 1 and 40 periods for spot procurement, PRC, MPC and QFC contracts and corresponding parameters

\begin{tabular}{lccc}
\hline Contract & One period & 40 periods & Parameters \\
\hline Spot & 1186 & $1.898 \times 10^{6}$ & - \\
PRC & 130 & $0.130 \times 10^{6}$ & $p_{r}=3.8$ \\
MPC & 452 & $0.723 \times 10^{6}$ & $q=10$ \\
QFC & - & $0.145 \times 10^{6}$ & $\alpha=1, \beta=448, \theta=0.1$ \\
\hline
\end{tabular}

